\title{
La réalisation des traitements optiques de surface
}

\author{
E. Pelletier
}

\begin{abstract}
Laboratoire d'Optique des Surfaces et des Couches Minces, Unité Associee au CNRS, Ecole Nationale Supérieure de Physique de Marseille, Domaine Universitaire de St Jérome, F-13397 Marseille cedex 13, France
\end{abstract}

Les traitements optiques de surface ont aujourd'hui une importance considérable car ce sont souvent eux qui conditionnent l'obtention des systèmes optiques les plus performants. Avec le développement des applications, on exige de plus en plus des traitements ayant des caractéristiques extrêmes ou très spéciales.

L'idéal serait de pouvoir modifier à volonté, grâce à ces traitements, les propriétés optiques d'une surface sans modifier ses propriétés géométriques.

Ceci pose des problèmes de calcul et des problèmes de réalisation. Il s'agit d'abord de concevoir la structure de l'empilement (on dit la formule) qui répond au problème posé. La réalisation doit être autant que possible conforme au calcul pour atteindre des performances optiques en accord avec les valeurs théoriques prévues. C'est la confrontation de la théorie avec l'expérience qui va nous permettre de progresser dans le développement des applications en réalisant des systèmes de couches de plus de plus complexes. Le but de ce cours est de donner une vue d'ensemble des difficultés. Cela nous permettra sur quelques exemples d'applications de mieux dégager les problèmes résolus ou à résoudre [1].

\section{I - LES TRAITEMENTS DE SURFACE. POURQUOI FAIRE?}

La conception des instruments optiques nous amène à nous intéresser à des surfaces de nature, de dimensions et de formes très diverses. Citons par exemple : un petit substrat plan de silice, un miroir de téléscope de huit mètres, l'extrémité d'une fibre optique, l'intérieur d'un tube capillaire ou d'une ampoule, un réseau de diffraction, une diode laser.... etc...etc. Les spécialistes de couches minces voudraient pouvoir proposer des traitements multicouches solides, résistants aux intempéries, pour conférer à ces surfaces, dans le domaine spectral utile de l'instrument, des propriétés optiques : facteur de réflexion $R(\lambda)$, facteur de transmission $T(\lambda)$, facteur d'absorption $\mathrm{A}(\lambda)$ (et le cas échéant, déphasage à la réflexion $\varphi(\lambda)$, déphasage à la transmission $\varphi^{\prime}(\lambda)$ ) qui répondent toutes à des conditions bien précises. On aurait là 
une des clés essentielles pour le développement de l'instrumentation et, si les coûts de fabrication ne sont pas prohibitifs, le nombre des applications que l'on pourrait envisager ne cesserait d'augmenter dans les années futures. Les filtres les plus courants ont des fonctions qui se classent dans les catégories suivantes:

- Antireflet

- Miroir à facteur de réflexion élevé, séparatrice de faisceaux

- Filtres passe haut ou passe bas.

- Filtres passe bande (bande large ou bande étroite)

- Polariseur (pour l'incidence oblique uniquement)

- Déphaseur

On va s'intéresser successivement aux méthodes de calcul des empilements puis aux problèmes de réalisation des couches : techniques de dépôt, choix des matériaux, mesure des épaisseurs, des indices, pour dresser un bilan des difficultés qui ont des importances variables selon les applications envisagées.

\section{II - LES PROBLEMES DE CALCUL}

\section{II-1) Analyse et synthèse}

Il faut disposer d'un modèle de couche pour calculer les propriétés optiques d'un empilement. Le modèle classique suppose que chaque couche est homogène, isotrope, à faces planes et parallèles. Dans ces conditions, deux paramètres suffisent du point de vue optique pour caractériser une couche mince. Ce sont l'indice de réfraction $\tilde{n}_{i}=n_{i}$. $i k_{i}$ et l'épaisseur $e_{i}\left(\tilde{n}_{i}\right.$ est un nombre complexe, fonction de la longueur d'onde; $n_{i}$ est appelé la partie réelle de l'indice et $\mathrm{k}_{\mathrm{i}}$ le coefficient d'extinction). On a donné en annexe un formulaire pour calculer assez facilement (à l'aide d'un micro-ordinateur) le facteur de réflexion $R$ et le facteur de transmission $T$ d'un empilement de couches minces. Le calcul de ces propriétés optiques se ramène à un produit de matrices, chaque couche étant représentée par une matrice $2 \times 2$.

On écrit $\mathrm{R}$ et $\mathrm{T}$ sous forme d'une fonction :

$$
R \text { et } T=f\left(\lambda, \theta \text { et } n_{0}, n_{s}\left(\tilde{n}_{i}, e i\right)_{i=1} \text { a } P\right) .
$$

$\rightarrow \quad \lambda$ et $\theta$ représentent les conditions d'éclairement : longueur d'onde et angle incidence dans le milieu ambiant (le cas échéant, on précisera l'état de polarisation).

$\rightarrow \quad \mathrm{n}_{0}, \mathrm{n}_{\mathrm{s}}$ sont les indices de milieu ambiant et du substrat. 
$\rightarrow \quad\left(\tilde{n}_{i}, e_{i}\right)_{i}=1$ à $P$ est la formule de l'empilement, le rang $i$ est compté à partir du substrat.

On notera ici que l'absorption est liée aux valeurs des indices de réfraction : si les coefficients d'extinction $\mathrm{k}_{\mathrm{i}}$ ne sont pas tous nuls alors $\mathrm{A} \neq 0$.

Sinon $\quad \mathrm{R}+\mathrm{T}=1$.

Le calcul sur ordinateur (que l'on appelle analyse) des propriétés optiques d'un empilement ne soulève pas de difficultés particulières. En vue des applications, le problème se pose différemment.

On a besoin, par exemple, d'un revêtement donnant à $\mathrm{R}$ des valeurs déterminées pour plusieurs longueurs d'onde $\lambda$ imposées. L'idéal serait de pouvoir déduire des valeurs de $\mathrm{R}$ et $\lambda$ le nombre de couches, le indices et les épaisseurs nécessaires pour obtenir ce résultat. Il n'y a pas de solution algébrique formelle pour résoudre ce problème de synthèse. On peut rapidement essayer de dresser un bilan sur ce problème du calcul préalable de la formule d'empilement.

\section{II-2) Principales techniques de synthèse [2]}

Certains empilements particuliers, systèmes périodiques, par exemple, présentent des propriétés relativement simples; si on accepte la "contrainte" d'utiliser de tels empilements, le calcul préalable se trouve facilité. On peut considérer le cas de contraintes sur les valeurs d'indices (correspondant à des matériaux existants) ainsi que sur les valeurs des épaisseurs optiques des couches. Le tableau ci-dessous résume les différents cas.

\begin{tabular}{|c|c|c|}
\hline indices $\mathrm{n}_{\mathrm{j}}$ & Empilements $\lambda_{0} / 4$ & $\begin{array}{l}\text { Epilements } \\
\text { "quelconques" }\end{array}$ \\
\hline $\begin{array}{l}\text { Matériaux diélectriques } \\
\text { disponibles }\end{array}$ & Empilements classiques & $\begin{array}{l}\text { Paramètres libres : } \\
\text { épaisseurs } \mathrm{e}_{\mathrm{i}} \text {. }\end{array}$ \\
\hline Indices intermédiaires & Relations de Pohlack & $\begin{array}{l}\text { Paramètres libres : } \\
\text { indices et épaisseurs }\end{array}$ \\
\hline
\end{tabular}

On ne peut ici donner que quelques remarques générales : 
$\rightarrow$ Couches quart d'onde

- C'est le cas des empilements constitués de couches d'épaisseurs optiques égales à une même valeur ne $=\lambda_{0} / 4$ ( $\lambda_{0}$ étant appelée la longueur d'onde de centrage). A la longueur d'onde particulière $\lambda=\lambda_{0}$, la matrice associée à chacune des couches prend une forme très simple. Pour le calcul des propriétés optiques en fonction de $\lambda$, POHLACK écrit le rapport $1 / \mathrm{T}$ sous la forme d'une série de Fourier avec le paramètre $\cos 1 \pi \frac{\lambda_{0}}{\lambda}$ où 1 varie de 0 à $P$ qui est le nombre de couches. Les coefficients de la série ne dépendent que des valeurs des indices des couches. Ce formalisme se prête bien à la synthèse de couches quart d'onde ou multiples de $\lambda_{0} / 4$ si les paramètres libres sont les indices des couches.

$\rightarrow \quad$ Couche équivalente. HERPIN a montré qu'un système symétrique de couches est équivalent à une couche unique d'indice de réfraction $n_{e}$ et d'épaisseur $e_{e}$, variables complexes en fonction de la longueur d'onde. THELEN [3] utilise systématiquement ce concept pour construire des empilements constitués de couches équivalentes. En outre, il montre comment on peut utiliser des couches équivalentes entre deux empilements pour adapter les impédances.

Le raisonnement est analogue à celui bien connu de l'antireflet qui adapte l'impédance entre un verre d'indice $\mathrm{n}_{\mathrm{v}}$ et l'air d'indice $\mathrm{n}_{\mathrm{a}}$ : il suffit d'une couche quart d'onde et d'indice $n_{c}=\sqrt{n_{v} n_{a}}$. Bien que les propriétés du "sous ensemble anti-reflet" ne soient valables que dans un domaine spectral restreint autour d'une longueur d'onde particulière, on dispose comme cela d'une solution approchée très utile. Cette technique de THELEN est certainement la méthode de synthèse qui est aujourd'hui la plus féconde.

$\rightarrow \quad$ Méthodes itératives. Partant d'un ensemble de couches, on calcule ses propriétés, leurs dérivées par rapport aux épaisseurs et au indices, puis on modifie ces paramètres en cherchant à se rapprocher d'une solution au problème posé, et on recommence. La puissance de calcul des ordinateurs modernes autorise l'emploi de cette méthode que l'on peut qualifier "de pêche à la ligne". L'amélioration suppose la définition d'un "coefficient de mérite" qui représente la distance entre les propriétés optiques désirées et celles calculées à chaque itération.

Le procédé est très imparfait : la solution n'est pas unique... Il présente toutefois de très nombreux avantages. On peut fixer les valeurs des indices et tenir compte de la dispersion en faisant appel à des matériaux d'emploi facile. On peut utiliser cette méthode de synthèse pour l'incidence normale, l'incidence oblique (avec deux états de 
polarisation) et même l'incidence variable. En outre, on introduit facilement dans le coefficient de mérite les tolérances admissibles sur les propriétés optiques recherchées et ceci permet souvent de conduire à terme le calcul de synthèse.

$\rightarrow$ Transformation de Fourier.

On a cherché à exploiter la relation de Fourier qui existe entre les propriétés optiques et un milieu inhomogène d'épaisseur finie. On arrive ainsi théoriquement à calculer un profil d'indice $\mathrm{n}(\mathrm{z})$. Mais on a des difficultés considérables à remplacer ce profil d'indice par un empilement équivalent de couches minces avec des valeurs d'épaisseurs et d'indices pour aboutir à une réalisation pratique.

Notons pour terminer l'existence de très nombreuses publications concernant des couches inhomogènes avec des lois de variation d'indice périodiques (ruggate filters). Encore une fois, la réalisation pratique de ce genre de filtres nous amène à envisager des systèmes complexes avec parfois près d'un millier de couches. Il s'agit encore d'études de laboratoire avant de pouvoir déboucher en production industrielle.

\section{II -3) Bilan et perspectives}

Même si le problème de la synthèse n'est pas un problème mathématique bien résolu, on n'en dispose pas moins d'un arsenal de techniques numériques plus ou moins empiriques pour apporter des solutions à des problèmes de filtrage si complexes soient-ils. Pour les applications, il faut aboutir à des solutions pratiques, c'est à dire à des formules d'empilements qui sont réalisables. Ceci implique que l'on ait pris le soin dans les calculs d'introduire les valeurs d'indices de couches que l'on sait réaliser et il nous faut surtout maintenant maîtriser le contrôle de l'épaisseur des couches.

\section{III - TECHNIQUES DE REALISATION DES COUCHES ET MICROSTRUCTURE.}

\section{III - 1) L'Evaporation sous vide}

Pour les applications optiques, les couches sont essentiellement produites par évaporation sous vide. Les matériaux utilisables pour donner des couches parfaitement transparentes de faible épaisseur sont relativement peu nombreux. Ce sont essentiellement des oxydes $\left(\mathrm{TiO}_{2}, \mathrm{SiO}_{2}, \ldots ..\right)$ des fluorures $\left(\mathrm{MgF}_{2}, \ldots.\right)$ ou des sulfures $(\mathrm{ZnS}, \ldots)$.

L'évaporation des matériaux ne va pas sans quelques difficultés. On utilise, soit des creusets chauffés par effet Joule, soit un bombardement électronique intense et 
correctement focalisé, pour atteindre des températures élevées et bien souvent le chauffage d'un matériau s'accompagne d'une décomposition chimique. Par exemple, la présence de sous-oxydes dans un creuset de dioxyde de titane $\left(\mathrm{TiO}_{2}\right)$ est malheureusement quasi inéluctable et on devra bien sûr s'inquiéter de la stoechiométrie des couches obtenues; elle conditionne la valeur de l'indice de réfraction.

Avec la précision qui nous intéresse, les problèmes spécifiques de la physique des matériaux en couches minces prennent une importance toute particulière. En effet, les études au microscope électronique montrent que les couches minces présentent une microstructure en forme de colonnes dont les diamètres sont de l'ordre de 10 à $30 \mathrm{~nm}$ et dont l'orientation des axes dépend de la direction du flux de matière évaporée.

De ce fait, il résulte que les propriétés physiques du matériau en couche mince sont systématiquement différentes de celles du matériau massif. C'est en particulier le cas de l'indice de réfraction et pour une couche mince, cette valeur va sensiblement dépendre des conditions physiques de l'atmosphère ambiante.

\section{III-2) Revue des techniques de réalisation de couches [4]}

Dans la mesure où l'état de la microstructure est responsable de limitations dans les performances optiques, il est évident que de nombreux travaux ont été consacrés à la résolution de cette difficulté. C'est la technique même de l'évaporation qui est remise en question; celle-ci risque bien d'ici quelques années de ne plus être la méthode reine des Opticiens. Ceci nous oblige à effectuer une revue sommaire des techniques de dépôt en insistant plus particulièrement sur celles qui paraissent aujourd'hui les plus prometteuses tout au moins pour certaines applications spécifiques. On va d'abord rappeler l'existence de deux grandes classes qui sont les méthodes chimiques et les méthodes physiques.

Les méthodes physiques comprennent les méthodes par évaporation ou éjection de matériau à partir d'une source : évaporation ou pulvérisation (Sputtering en Anglais). Les méthodes chimiques dépendent d'une réaction chimique spécifique avec une séparation électrique des ions (électrolyse, anodisation) ou un effet thermique (dépôt en phase vapeur). La frontière entre ces deux classes, physique et chimique, n'est pas nettement tracée. 


\section{Methodes chimiques}

Sauf de rares exceptions, les méthodes chimiques sont peu utilisées en optique. Ceci s'explique souvent par le fait que les substrats de l'optique ne supportent pas des traitements thermiques poussés.

Un exemple: Dépôt de solution (dip coating). Il suffit de tremper le substrat dans un liquide où de déposer une goutte sur la surface et de l'étaler en faisant tourner le substrat (Spin coating). On prendra par exemple du diethyl-dichloro titane pour avoir de l'oxyde de titane de bonne qualité ! Ces méthodes ont un intérêt considérable pour les traitements des optiques des lasers de puissance. Malgré leur fragilité mécanique, ils résistent très bien aux flux lumineux intenses.

\section{Methodes physiques}

Les techniques en phase vapeur s'articulent en trois étapes :

i - Génération de vapeur par une simple évaporation et sublimation ou par une pulvérisation cathodique.

ii - Transport du matériau sous forme de vapeur de la source vers le substrat.

iii - Condensation sur le substrat

Les difficultés déjà signalées avec l'évaporation viennent du fait que l'énergie au niveau des atomes volatilisés est de l'ordre de $0,1 \mathrm{eV}$; bien qu'ils perdent peu d'énergie à la traversée de l'enceinte à vide, on a une nucléation assez hétérogène. Il faut augmenter sensiblement cette énergie pour assurer la formation d'un dépôt plus compact.

On retiendra quelques techniques intéressantes pour l'optique:

(a) L'évaporation en atmosphère réactive (avec une température limite qui dépend du type de substrat chauffé)

(b) L'évaporation assistée par faisceau d'ions (ion assisted deposition ou I.A.D.). Il s'agit d'une évaporation classique complétée par un canon à ions qui bombarde le matériau qui se condense sur le substrat.

(c) La pulvérisation cathodique entre plateaux d'un condensateur. Pour éviter une saturation de charge entre anode et cathode, la pulvérisation haute fréquence est particulièrement intéressante.

(d) L'Ion Plating (BALZERS) ou META MODE (OCLI). C'est une technique intéressante entre l'évaporation et la pulvérisation. Le porte-substrats est isolé électriquement et les substrats se trouvent chargés négativement pour avoir un champ électrique entre la source et les substrats. L'évaporation est réalisée en présence d'une atmosphère d'argon (ou d'oxygène) de l'ordre de $10^{-2}$ Torr c'est à dire dans un plasma. 
On essaye évidemment d'arranger les conditions du plasma pour optimiser les paramètres au niveau des trois étapes $i$, ii et iii ci-dessus.

(e) Pulvérisation par faisceau d'ions (ion beam sputtering ou I.B.S. et dual ion beam sputtering ou D.I.B.D.S.). Un canon à ions est utilisé pour pulvériser la cible, le cas échéant un second canon à ions sert à compacter le matériau qui se condense sur le substrat.

Il faut surtout retenir de cet éventail de techniques qu'il n'y a pas de méthode miracle. Chacune d'elles a ses avantages et ses inconvénients. En production, on s'intéresse à l'état de la microstructure des dépôts mais la notion des coûts de revient et les problèmes liés à la dimension des surfaces à traiter jouent également un rôle essentiel. L'industrie de l'optique doit évoluer et on ne va pas vers une simplification des moyens à mettre en œuvre pour obtenir des couches de qualité !

\section{III - 3) Les matériaux, leurs indices}

On utilise évidemment les métaux pour des miroirs de qualité courante : Aluminium, Argent dans le visible, Chrome, Or dans l'infrarouge, en particulier à 10 $\mu \mathrm{m}$.

En ce qui concerne les matériaux transparents diélectriques ou semiconducteurs, la liste des matériaux utilisables diffère évidemment d'une technique de dépôt à l'autre. Avec les techniques les plus classiques comme l'évaporation, MACLEOD donne dans son livre [1] la liste d'une cinquantaine de matériaux. On a vu que la plupart des matériaux utilisables dans le visible sont des sulfures, des fluorures et surtout des oxydes. Pour un bon nombre d'entre eux, la zone de transparence se limite à l'intervalle $400 \mathrm{~nm}-2 \mu \mathrm{m}$ et ils ne sont donc pas utilisables pour des traitements dans l'infrarouge proche et lointain. Grosso modo les valeurs d'indices dans le visible sont toutes comprises entre 1,35 et 2,4 (Fig. 1).

Pour l'infrarouge on a heureusement quelques matériaux intéressants mais ceux-ci ne sont pas transparents dans le visible; c'est le cas du germanium (Ge, $\mathrm{n}=$ $4,2)$ du tellurure de plomb $(\mathrm{PbTe}, \mathrm{n}=5,5)$, du silicium $(\mathrm{Si}, \mathrm{n}=3,5) \ldots$

Bien évidemment, les valeurs de l'indice données ici ne constituent qu'une indication. On a besoin de connaître avec précision les valeurs de $n(\lambda)$ et $\mathrm{k}(\lambda)$ pour les couches que nous fabriquons avec notre appareillage, en respectant dans les moindres détails tout un ensemble de conditions pour essayer de garantir d'un dépôt à l'autre une bonne répétabilité de la production. On décrira plus loin les méthodes utilisées pour déterminer ces valeurs des constantes optiques. 


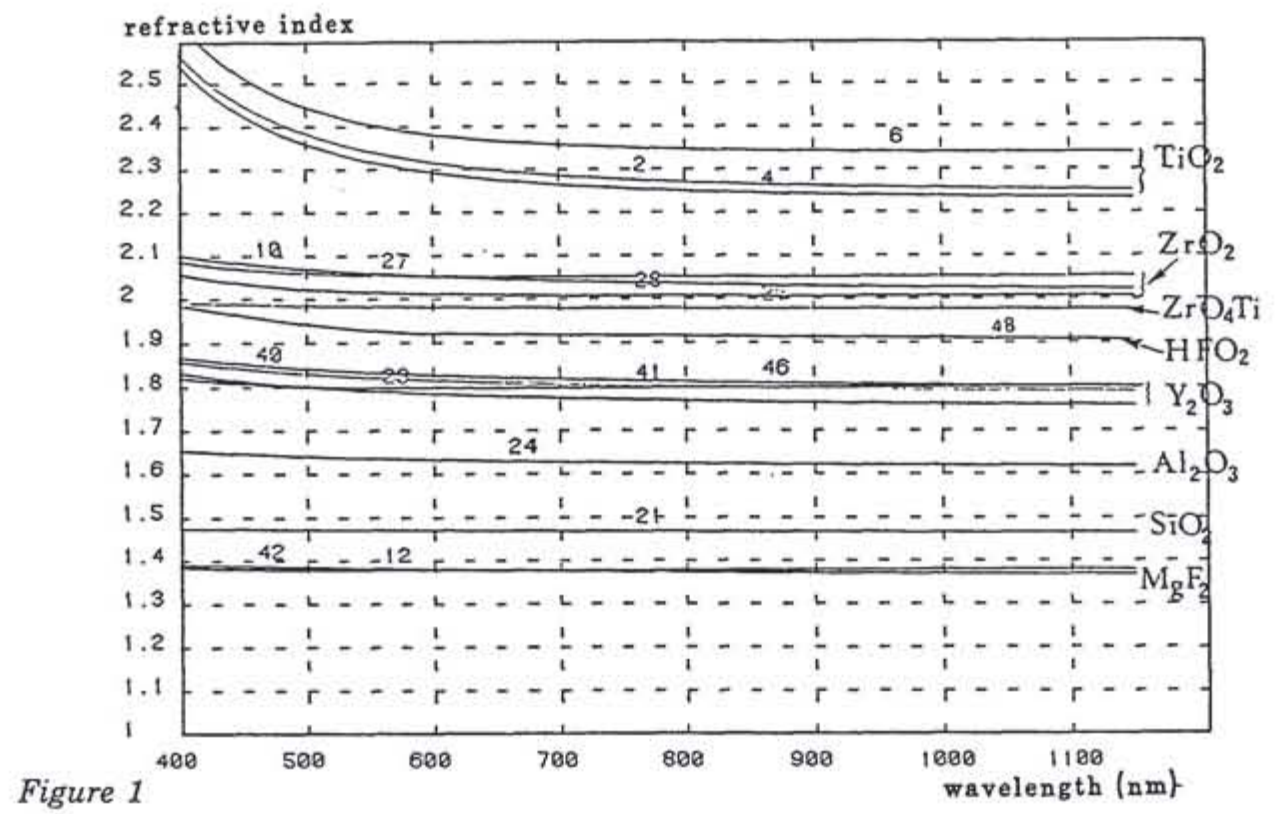

\section{III - 4) L'uniformité des dépôts}

Considérons une source (plus ou moins large selon les cas) et une surface (de forme à priori quelconque) qu'il s'agit de traiter. Quelle uniformité peut-on espérer obtenir ? Cela dépend beaucoup des caractéristiques d'émission de la source et des conditions géométriques : distance relative des différents éléments. Dieu le Père a montré que l'on avait intérêt à faire tourner la Terre par rapport au Soleil pour améliorer l'uniformité de l'éclairement (il s'agit d'une valeur moyenne prise sur un intervalle de temps relativement grand par rapport au temps pris pour une rotation). En plus de la rotation, un mouvement planétaire arrange encore la situation. Le problème est posé, on peut trouver dans la littérature des équations [1], il faut aussi apporter des solutions pratiques adaptées à chaque type d'appareillage et qui tiennent compte de la taille des substrats.

A titre d'exemple, la Fig. 2 montre la distribution théorique d'épaisseur que l'on peut espérer obtenir pour une distance h entre source et substrat, la source étant décalée par rapport à l'axe du substrat d'une distance $R$.

Remarquons pour terminer que l'on devra aussi s'inquiéter de l'uniformité de la valeur de l'indice sur la surface traitée. 
D'après: "tHiN-FilM OPtiCAl Filters" (Macleod - 1969)

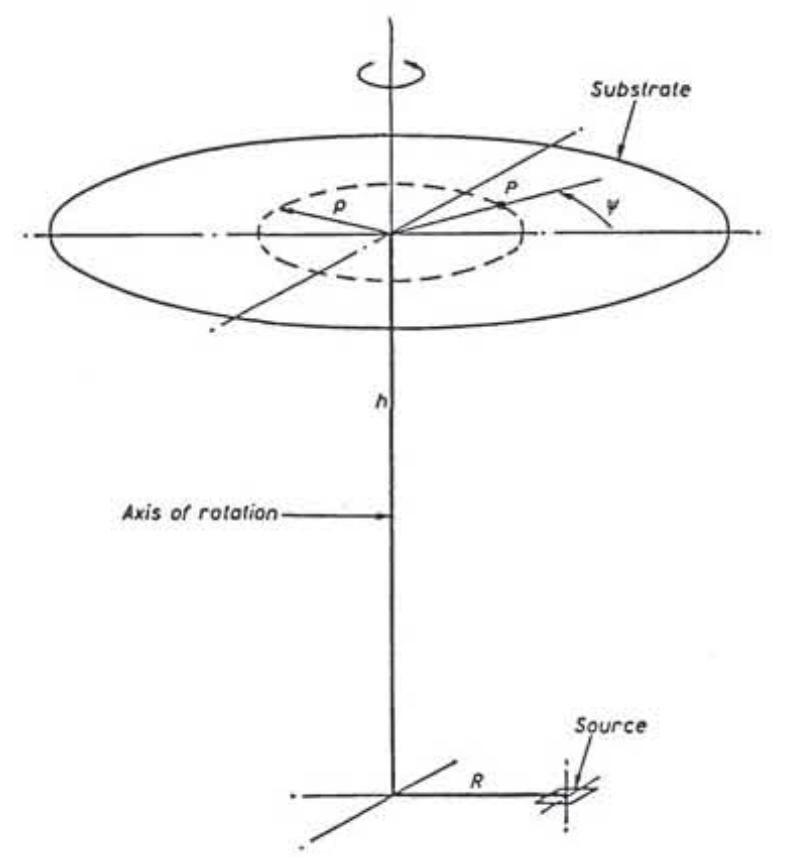

Figure 2

Diagram showing the geometry of the evaporation from a stationary offset source onto a rotating substrate.

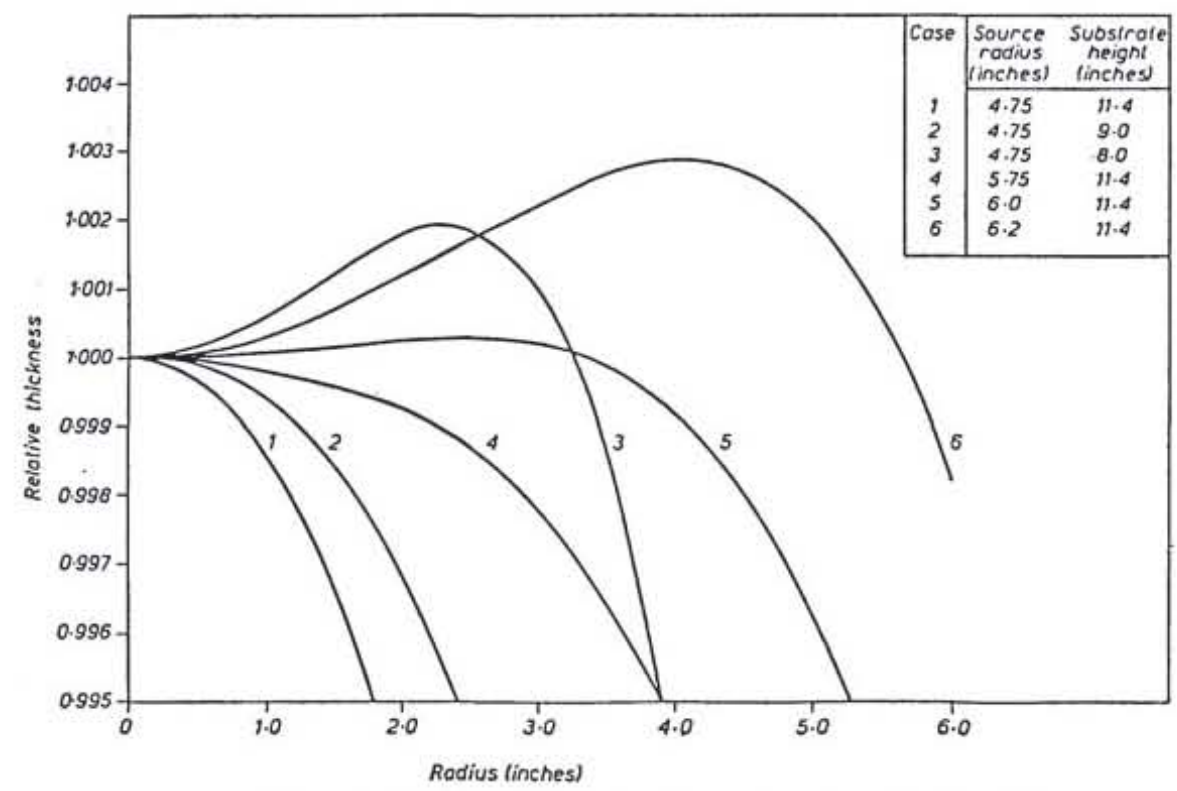

Theoretical film thickness distribution on substrates rotated about the centre ot the plant for various source radii and substrate heights. The sources are assumed to be small directed surfaces parallel to the substrates. 


\section{III - 5) Questions annexes}

On se limitera à quelques banalités qui risquent de paraître évidentes, sauf peutêtre au spécialiste qui doit se débrouiller avec ces problèmes.

Dans un empilement, les couches "couchent ensemble"; il est important qu'elles se supportent, si on veut qu'elles vieillissent bien.

On aura des exigences en ce qui concerne la résistance à l'abrasion des traitements : c'est essentiel pour les hublots des véhicules, la lunetterie. Mais tout bêtement, il y aussi des problèmes d'adhésion des couches sur le substrat. Peu de gens apprécient le spectacle fascinant que nous offrent des couches en train de se soulever et de se détacher peu à peu du substrat.

Enfin, on ne peut pas oublier le fait que parfois les utilisateurs de couches ont des idées bizarres et qu'ils soumettent ainsi nos pauvres traitements aux pires humiliations : bain prolongé dans des liquides plus ou moins caustiques, exposition au froid, à la pluie... ou aux rigueurs du vide interstellaire.

On accordera une mention spéciale à ceux qui préferent les expositions intenses aux flux laser. Il faut dire que dans une cavité laser (c'est à dire un Fabry-Perot) la quantité maximale d'énergie qui peut être emmagasinée dépend pour l'essentiel de la qualité des miroirs : ce sont eux qui servent de fusibles ! Les motivations des recherches dans ce domaine sont donc très fortes.

\section{IV - PROPRIETES OPTIQUES ET TOLERANCES DE REALISATION}

\section{IV - 1) Performances optiques}

La synthèse permet de calculer les caractéristiques (nombre de couches, indices, épaisseurs) souhaitables pour obtenir des propriétés optiques données. Les indices de réfraction des couches simples varient facilement de quelques pour-cent en fonction de paramètres difficilement contrôlables : pression, nature des gaz résiduels, température, etc... Il est évidemment difficile de contrôler les épaisseurs optiques déposées avec une précision de quelques Angströms. De toute façon on ne peut éviter une certaine imprécision; de plus l'absorption, les irrégularités superficielles ne sont jamais strictement nulles. La réalisation peut donc s'écarter notablement du projet. Ceci nous amène à nous poser un certain nombre de questions :

(1) Pour réaliser un empilement donné, quelle est la meilleure méthode (optique) de contrôle pour chacune des couches successives? 
(2) Avec quelle précision les données établies par le calcul doivent-elles être respectées?

(3) Comment les écarts de réalisation influenceront-ils les résultats obtenus ?

(4) Dans un système de couches calculé, y a t-il un point critique sur lequel une précision particulièrement élevée est requise ? On sait par exemple que dans un FabryPerot, l'épaisseur de la couche médiane est critique pour la localisation da la banque passante.

Pour la facilité de la présentation, nous examinerons d'abord le point (3) qui est le problème du calcul des tolérances de réalisation. On ne confondra pas les points de vue de l'utilisateur et du fabricant.

On veut obtenir un filtre ayant des caractéristiques spectrales précises, par exemple un profil $\mathrm{T}(\lambda)$ dans un domaine de longueurs d'onde déterminé pour une incidence bien définie. Il est commode de représenter les tolérances sur T par un gabarit (Fig.3) qui encadre le profil idéal $\mathrm{T}_{\text {idéal. }}$ On exige que le filtre reste dans les limites prescrites (zone non hachurée).

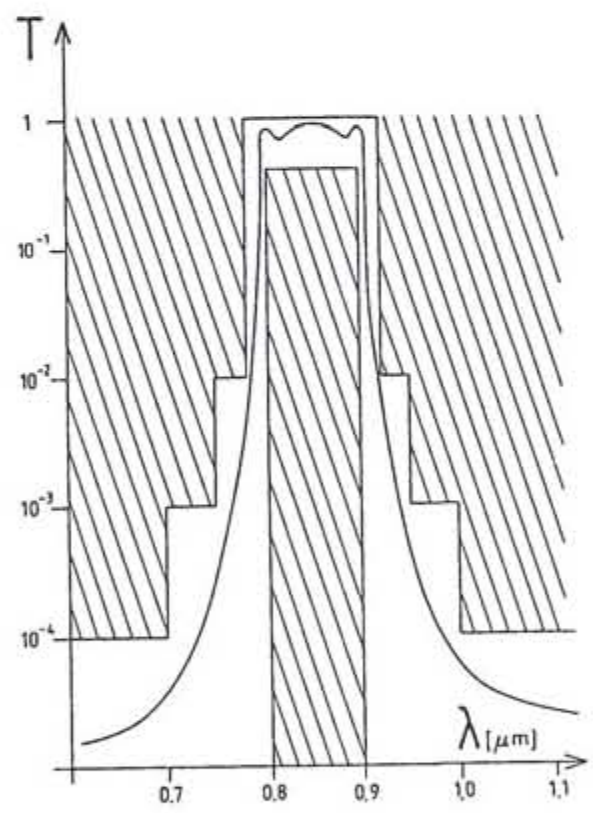

Figure 3
La première étape dans la conception du filtre est la synthèse. On a défini un coefficient de mérite qui représente la distance entre le profil calculé à l'itération $\mathrm{k}$ et le profil désiré.

$$
f_{k}=\left\|T_{\text {idéal }}(\lambda)-T_{k}(\lambda)\right\|
$$

On aboutit à une solution acceptable. Il s'agit d'un profil $\mathrm{T}_{\text {th }}$ qui théoriquement est obtenu avec

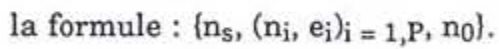

C'est ce profil $\mathrm{T}_{\text {th }}$ qui va nous servir de profil de référence pour le développement de l'expérimentation. On l'appelera le profil parfait : $\mathrm{T}_{\text {parf }}$. 
La réalisation va différer du projet ; on souhaite évidemment que cette différence soit aussi réduite que possible et ceci risque de nécessiter une étude de mise au point. On va à nouveau utiliser le coefficient de mérite pour caractériser la qualité du résultat expérimental $\mathrm{T}_{\text {exp. }}$

Le fabricant de l'empilement s'intéressera à la distance:

$$
f^{*} \exp =\left\|T_{\text {parf }}-T_{\exp }\right\|
$$

qu'il cherchera à réduire pour améliorer sa production et garantir une bonne répétabilité malgré des rendements intensifs (problèmes d'uniformité des dépôts).

Pour répondre à la demande de l'utilisateur c'est toutefois la distance suivante qui importe :

$$
\mathrm{f}^{*} \exp =\left\|\mathrm{T}_{\text {ideal }}-\mathrm{T}_{\exp }\right\|
$$

On tiendra évidemment compte des tolérances fixées sur T idéal pour conclure cette étude.

Connaissant les tolérances sur $\mathrm{T}$, il faudrait remonter à une estimation des tolérances sur la formule de l'empilement qu'il s'agit de réaliser. On est en droit d'admettre ici qu'il n'y a pas de difficultés avec les valeurs des indices des milieux extrêmes $n_{s}$ et $n_{0}$ et avec le nombre de couches $P$. Le problème du calcul des tolérances se réduit donc aux $2 \mathrm{P}$ paramètres relatifs aux couches constituant l'empilement. Malheureusement, ce problème n'est pas facile à résoudre et ceci tient à de nombreuses raisons qu'il faut examiner grâce aux approches successives que l'on peut mener.

\section{IV - 2) Etude des tolérances}

\section{1) Cas d'une couche isolée}

L'idée la plus simple consiste à considérer une erreur isolée portant soit sur $\mathrm{n}$, soit sur e, et de calculer les conséquences de cette erreur sur les propriétés optiques de l'empilement.

Ce genre de calcul permet de déceler les couches critiques de l'empilement, couches pour lesquelles une précision particulièrement poussée sera nécessaire.

Un exemple classique est celui d'un filtre Fabry-Perot de formule : verre (HB HB...HB) $2 \mathrm{H}(\mathrm{BH} . . . \mathrm{H})$ air 
où $\mathrm{H}$ et $\mathrm{B}$ représentent respectivement des couches de haut et bas indice d'épaisseur optique quart d'onde pour une longueur d'onde $\lambda_{0}$. C'est à dire $\mathrm{n}_{\mathrm{H}} \mathrm{e}_{\mathrm{H}}=\mathrm{n}_{\mathrm{B}} \mathrm{e}_{\mathrm{B}}=\lambda \alpha_{0} / 4$.

Le calcul montre que la moindre erreur de réalisation sur la couche médiane $\delta e_{H}$ amène un décalage sur le positionnement de la bande. Pratiquement ce décalage est de $\left(\lambda-\lambda_{0}\right)=\frac{\delta e_{H}}{e_{H}} \lambda_{0}$.

En comparaison, les tolérances sont plus lâches sur les couches des miroirs et ce d'autant plus que la couche perturbée est éloignée de la couche médiane.

\section{2) Cas d'erreurs multiples}

Dans la pratique, on n'a évidemment pas affaire à une erreur isolée. Chacune des couches de l'empilement est affectée par des erreurs de réalisation. Vu le nombre de configurations qu'il s'agit d'explorer, le calcul devient vite impossible et on ne peut que se contenter de l'analyse de quelques cas particuliers.

Pour avoir une idée des tolérances, il faut faire appel à des méthodes statistiques. On va admettre que chaque couche est affectée d'une erreur : $\delta \mathrm{e}_{\mathrm{i}}$ et résulte d'un tirage aléatoire fait sur une Gaussienne de valeur moyenne $e_{\mathrm{i}}$ et de largeur $\delta \sigma_{\mathrm{e}}$.

De même, on peut introduire l'existence d'une erreur sur $\mathrm{n}$ avec distribution Gaussienne (largeur $\delta \sigma_{n}$ ). Et on pourra examiner à volonté l'effet d'erreurs simultanées sur $\mathrm{n}$ et e.

Il nous appartient de "fixer de façon raisonnable" des valeurs de $\delta \sigma_{e}$ et $\delta \sigma_{n}$ pour avoir une bonne représentation des conditions expérimentales. On pourra alors procéder à une série de tirages et aboutir à une représentation globale des résultats de ces différents calculs en traçant, sur la même figure, le résultat de chacun des profils spectraux obtenus. On retiendra deux cas extrêmes.

a) Chaque profil est très différent des autres et on sort quasi systématiquement du gabarit : on a fort peu de chances d'arriver à réaliser un filtre correct !

b) Le second cas est un cas idéal. En dépit des erreurs aléatoires sur e (et n), les profils restent collés les uns aux autres, tout au moins dans un domaine spectral étendu par rapport à celui qui nous intéresse. La réalisation ne devrait donc pas soulever de difficultés majeures.

\section{3) Notion d'erreurs indépendantes et "d'erreurs liées"}

On sait qu'en effectuant un contrôle optique pour une longueur d'onde $\lambda_{0}$ déterminée, on accède à une mesure de l'épaisseur optique $n_{\lambda_{0}}$ e de la couche en cours de formation. On ne peut donc pas considérer $\mathrm{n}$ et e indépendamment du point de vue 
des erreurs. Les distributions statistiques doivent porter sur $\mathrm{n}$ d'une part, et le produit ne d'autre part.

Ce n'est pas la seule remarque. La mesure de l'épaisseur optique découle d'une mesure des propriétés optiques; on contrôle par exemple l'évolution du facteur de transmission au cours du dépôt. Notons qu'il n'y pas une relation simple entre ces variations de transmission et des variations d'épaisseur.

Au lieu de considérer les incertitudes sur les caractéristiques optogéométriques des couches, il faut plutôt arriver à chiffrer les conséquences d'une erreur de mesure sur les propriétés optiques. Tout compte fait c'est l'incertitude sur le repérage du critère d'arrêt qui va conditionner la précision. La couche qui est ainsi réalisée a une épaisseur plus ou moins différente de celle désirée et ceci dépend de la sensibilité de la technique de contrôle.

Au niveau des épaisseurs, les incertitudes dépendent du rang de la couche. On ne peut pas conserver l'hypothèse d'une répartition gaussienne $\delta \sigma_{e}$ identique pour toutes les couches de l'empilement. On doit prendre des distributions avec des $\delta \sigma_{e}$, fonction du rang $i$, pour être adaptées à la sensibilité de la technique de contrôle utilisée.

Il y a plus grave encore : lorsque l'on procède à un contrôle optique direct, c'est à dire lorsqu'on effectue le contrôle de la totalité de l'empilement sur le même substrat, on va voir un processus d'erreurs cumulatives. En effet, la variation de transmission qui est observée au cours du dépôt d'une couche dépend évidemment de l'épaisseur $\mathrm{e}_{\mathrm{j}}$ de cette couche mais elle dépend également des caractéristiques (épaisseurs et indices) des couches précédemment déposées. Les erreurs de réalisation ont pour effet de perturber le contrôle optique et, au fur et à mesure de la construction de l'empilement, les effets de ces erreurs s'accumulent. L'expérience montre que l'on ne peut pas négliger ce phénomène si on veut aboutir à une estimation correcte des tolérances de réalisation.

SEULES LES TECHNIQUES DE SIMULATION DU CONTROLE VONT PERMETTRE de conduire une approche correcte du CALCUL DES TOLERANCES DE REALISATION. Les épaisseurs optiques (et par conséquent les erreurs) sont liées les unes aux autres; et la relation qui définit les liens dépend entièrement de la technique de contrôle utilisée. Nous reviendrons plus loin sur l'exploitation des calculs de simulation.

\section{V- CONTROLE DE LA FORMATION DES EMPILEMENTS}

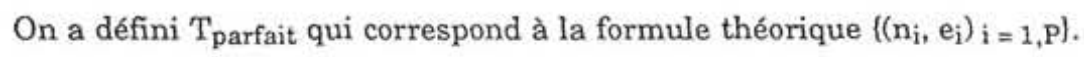


Durant la formation il faut en principe contrôler en détail les valeurs des indices et les épaisseurs.

\section{V - 1) Méthodes de contrôle}

$\mathrm{Au}$ fur et à mesure de la formation, il est possible de contrôler :

1) La masse de matériau qui vient se condenser sur un substrat particulier grâce à un quartz piézoélectrique. La variation de fréquence est grosso modo proportionnelle à la masse de la couche déposée.

Si les problèmes de calibrage sont bien résolus, le quartz est très utile pour reproduire une expérience.

2) L'évolution des propriétés optiques mesurées sur un substrat particulier (verre de contrôle).

On peut mesurer le facteur de transmission ou de réflexion pour des conditions d'éclairement (incidence normale par exemple) sur un domaine spectral plus ou moins étendu : monochromatique ou large bande.

Avec un contrôle optique on peut envisager deux cas extrêmes :

- le contrôle optique direct, c'est à dire que l'on contrôle la totalité de l'empilement sur le substrat à traiter qui nous intéresse.

- le contrôle optique indirect : on utilise des verres témoins. On peut utiliser autant de témoins que l'on veut, par exemple un par couche, pour mesurer l'indice de la couche, suivre l'augmentation d'épaisseur et arrêter le dépôt lorsque l'on atteint la valeur requise.

\section{V-2) Contrôle optique}

La mise en œuvre d'un système de contrôle sur un bâti de dépôt nous amène à répondre à la série de questions suivantes :

- Faut-il utiliser un contrôle direct ou un contrôle indirect ?

- Doit-on mesurer le facteur de transmission ou le facteur de réflexion? Ou vautil mieux prévoir une mesure ellipsométrique?

- Peut-on se contenter de mesures relatives, ou faut-il mettre en œuvre des mesures absolues ? et ce, pour quelles longueurs d'onde?

On commencera par l'étude des problèmes posés par le contrôle d'une couche unique avant de s'intéresser à la généralisation pour un empilement. Un contrôle indirect avec autant de témoins que de couches se ramène évidemment au cas du contrôle d'une couche unique. 


\section{V-3) Couche unique}

L'objectif est de réaliser une couche de caractéristiques données $\left(\mathrm{n}_{1}, \mathrm{e}_{1}\right)$ sur un substrat $\mathrm{n}_{\mathrm{s}}$. Pour effectuer le contrôle, on a deux étapes qui sont le calcul préalable et la mesure au cours du dépôt pour arrêter la formation de la couche à la valeur voulue.

La Fig. 4 montre le résultat du calcul de la transmission sur un large domaine spectral, lorsque l'épaisseur varie de 0 (cas du substrat nu) à l'épaisseur requise $e_{1}$. Pour ce calcul, il faut connaître la valeur de l'indice de réfraction : nombre complexe $\overline{\mathbf{n}}=\mathbf{n} \cdot \mathbf{i k}$, fonction de la longueur d'onde dans les conditions de dépôt de la couche. Le profil $\mathrm{T}^{\text {th }}$ 1 pour la formule $\left\{\mathrm{n}_{\mathrm{s}},\left(\mathrm{n}_{1}, \mathrm{e}_{1}\right) \mathrm{n}_{0}\right\}$ est donc le profil sous vide.

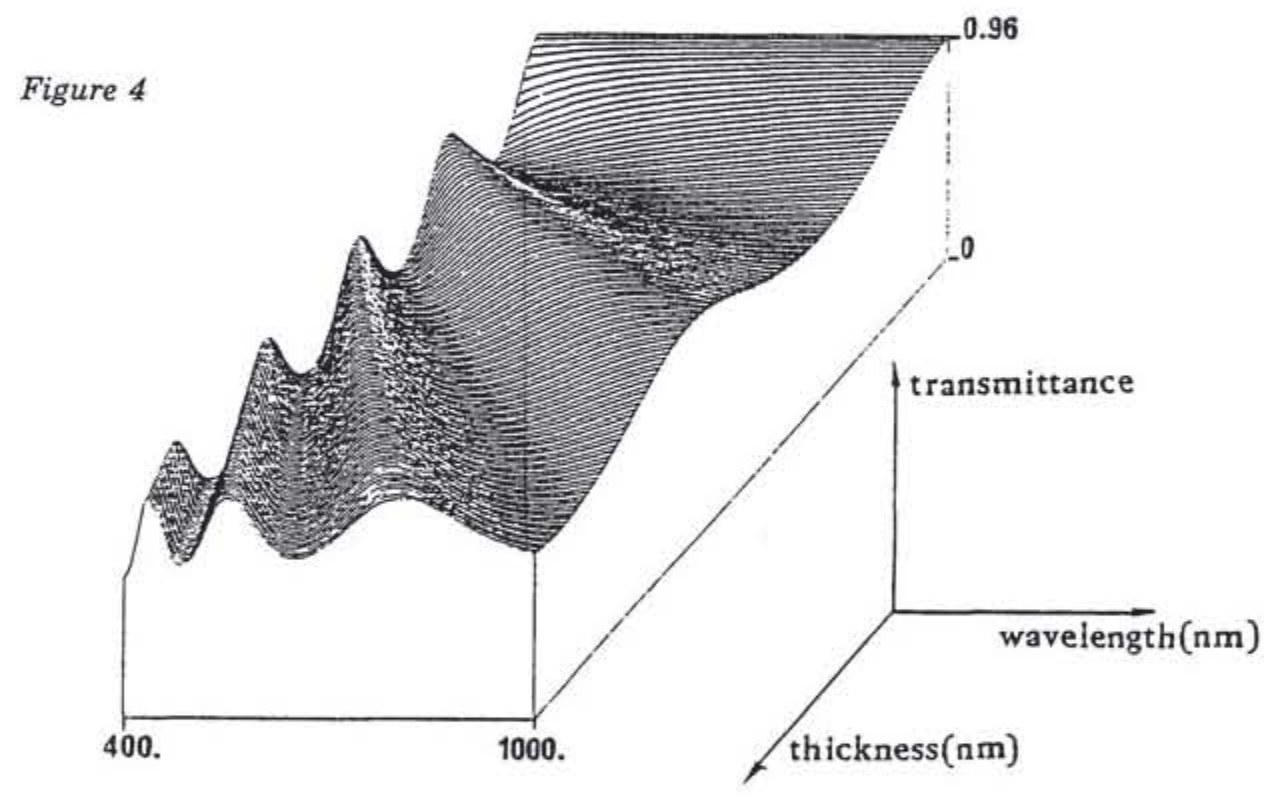

Il faut que la mesure au cours du dépôt permette de repérer l'instant précis pour lequel la couche atteint l'épaisseur $\mathrm{e}_{1}$. On a deux options:l'une consiste à se limiter à une mesure monochromatique; pour l'autre on effectuera des mesures sur une large bande spectrale. Le tableau ci-dessous résume les différents choix possibles pour le critère d'arrêt.

\begin{tabular}{|l|l|l|}
\hline & Mesures relatives & Mesures absolues \\
\hline Monochromatique & Repérage d'extremum & Valeur calculée de $\mathrm{T}$ \\
\hline Large bande & & Profil calculé T $(\lambda)$ \\
\hline
\end{tabular}


Il est toujours difficile de réaliser des mesures absolues du facteur de transmission $\mathrm{T}$ parce qu'il faut calibrer les mesures de flux grâce à des valeurs de référence (la transmission du substrat nu et le zéro de transmission par exemple). Il faut atteindre une précision d'un demi pour-cent sur la mesure de $\mathrm{T}$ pour une longueur d'onde si on veut avoir une incertitude relative sur l'épaisseur optique qui ne dépasse pas $2 \%$ dans le meilleur des cas. Encore faut-il pour cela bien choisir la longueur d'onde de contrôle afin de bénéficier de la meilleure sensibilité. On améliore sensiblement la précision avec un contrôle simultané sur une série de longueurs d'onde dans une large bande spectrale, mais l'appareillage de contrôle est loin d'être d'emploi facile. On préfere souvent les mesures relatives, il faut alors choisir la longueur d'onde de contrôle $\Lambda$, pour avoir un extremum de transmission pour l'épaisseur optique voulue : $\mathrm{n}_{1}\left(\Lambda_{1}\right) e_{1}=\mathrm{k} \Lambda_{1} / 4$ avec $\mathrm{k}$ entier $(\mathrm{k}=1,2,3 \ldots .$.$) . On sait$ toutefois qu'il est difficile d'arrêter le dépôt sur un extremum et la moindre erreur de pointé (Fig. 5) entraîne vite une erreur importante sur la valeur de ne. Par exemple, avec une couche d'indice 2,3 un dépassement de $\Delta \mathrm{T}=0,005$ amène à une épaisseur optique de $1,12 \frac{\Lambda_{1}}{4}$ au lieu de $\frac{\Lambda_{1}}{4}$.

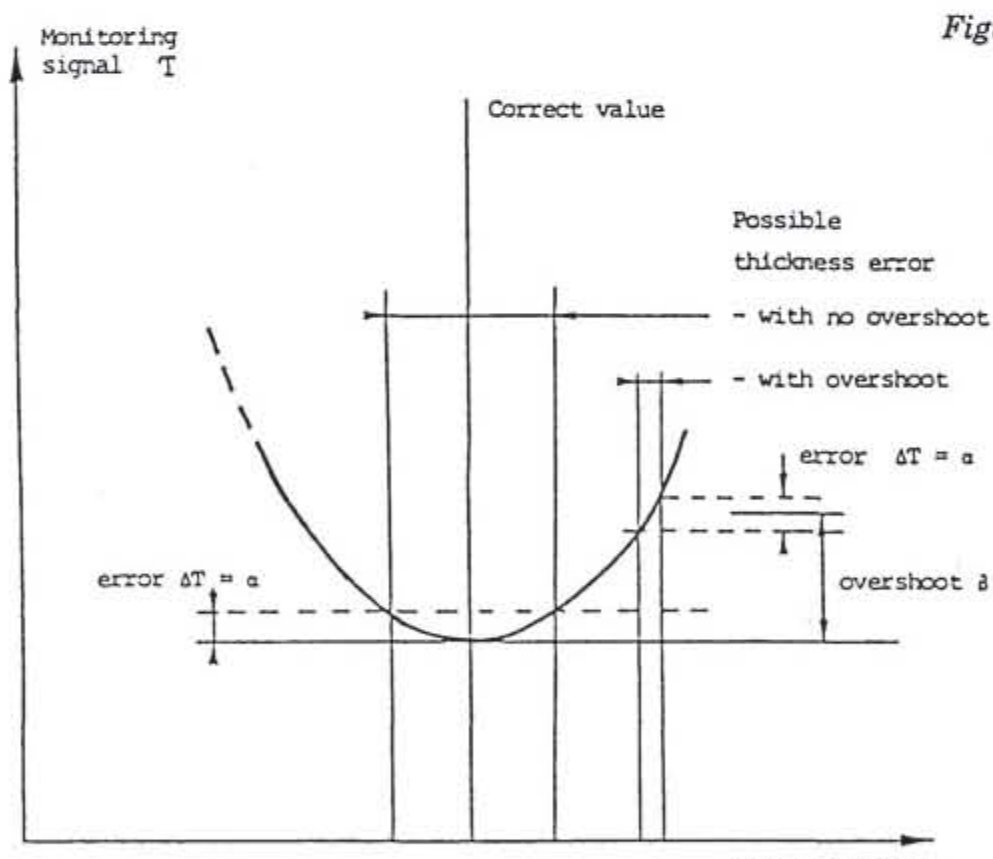

Figure 5

Layer thickness 
Les techniques de dérivation sont très utiles pour améliorer la qualité du pointé de l'extremum. Encore faut-il les employer correctement. La transmission est une fonction de deux variables qui sont $t$ et $\lambda$ c'est à dire le temps (ou l'épaisseur e) et la longueur d'onde.

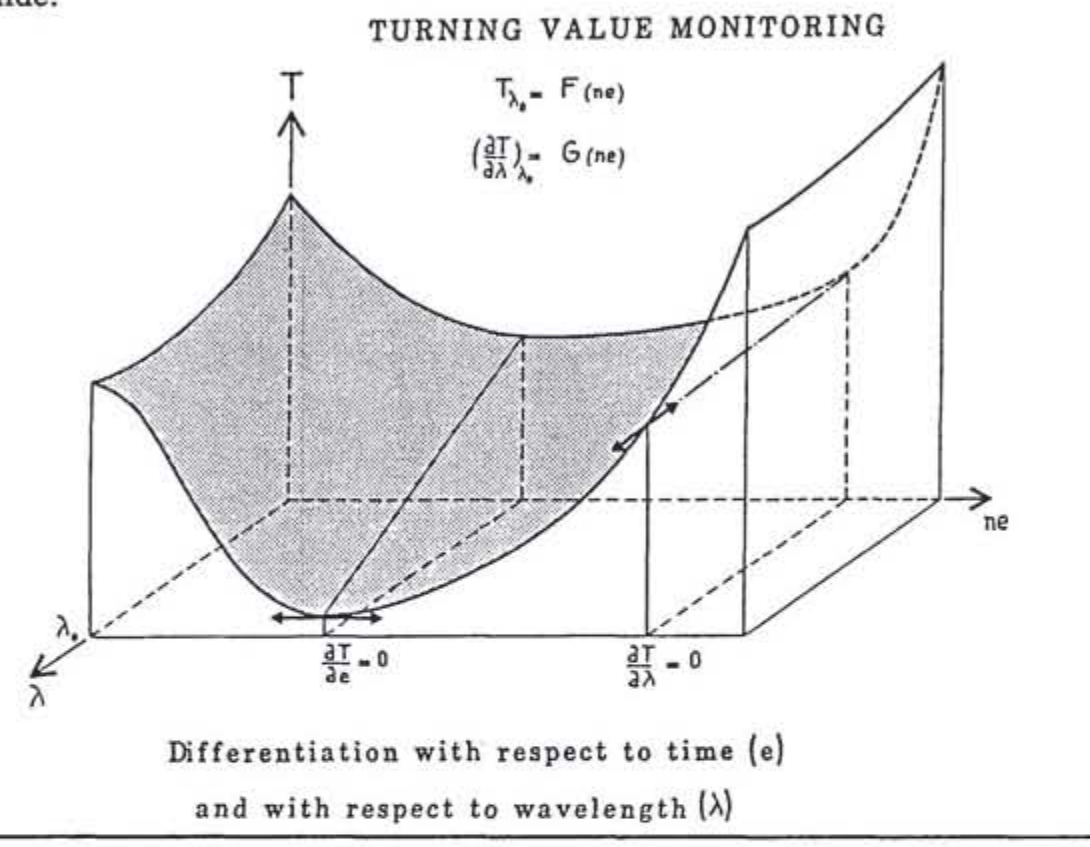

La Fig. 6 peut aider le lecteur à éviter toute confusion entre les deux dérivées partielles $\frac{\partial T}{\partial \lambda}$ et $\frac{\partial T}{\partial e}$. Exception faite de quelques cas très particuliers, les zéros de ces deux dérivées ne coïncident pas. C'est le calcul préalable sur ordi-nateur qui va nous permettre d'exploiter pleinement une tech-nique de contrôle avec les dérivées.

Le principe de l'appareillage de mesure est facile à décrire. On utilise une source de lumière blanche pour former un faisceau qui traverse le verre témoin et qui est récupéré à la sortie de l'enceinte par un monochromateur. La fente de sortie du monochromateur est équipée d'un récepteur multicanal comme par exemple une barrette de 1024 photodiodes. Chaque diode reçoit une longueur d'onde particulière $\lambda_{\mathrm{j}}, \mathrm{j}$ étant le rang de la diode. Elle permet la mesure d'un flux proportionnel à $T\left(\lambda_{j}\right)$ et qui, pendant le dépôt d'une couche, varie au cours du temps. L'accès aux deux dérivées est immédiat :

- On relève à intervalles de temps réguliers le signal $T\left(\lambda_{j}, t\right)$ et le calcul de la différence entre ces signaux donne DTE qui représente la dérivée par rapport au temps. 
- De part et d'autre de la diode $j$, on extrait les valeurs de $T\left(\lambda_{j-1}\right)$ et $T\left(\lambda_{j+1}\right)$ et la différence donne la dérivée par rapport à la longueur d'onde (signal DTL).

La Fig. 7 donne un exemple d'enregistrement des dérivées DTE et DTL recueillies au cours du temps lors du dépôt d'une couche d'épaisseur optique $3 \frac{\lambda_{j}}{4}\left(\lambda_{j}=\right.$ $720 \mathrm{~nm}$ ). Pour ce matériau et à cette longueur d'onde, l'indice de réfraction présente une dispersion d'indice très faible $\left(\frac{\mathrm{dn}}{\mathrm{d} \lambda}\right)=0$ et dans ces conditions les zéros de DTE et de DTL sont confondus. Chacun des zéros de DTE correspond à une épaisseur optique de $\frac{\lambda_{\mathrm{i}}}{4}, \frac{2 \lambda_{\mathrm{i}}}{4}$ et $\frac{3}{4} \lambda_{\mathrm{j}}$.

Pour les zéros de DTL cette relation n'est pas exactement vérifiée si l'indice est dispersif.

Le tableau donne un bref résumé pour comparer l'intérêt respectif des deux dérivées en tant que critères de contrôle de la formation d'une couche.

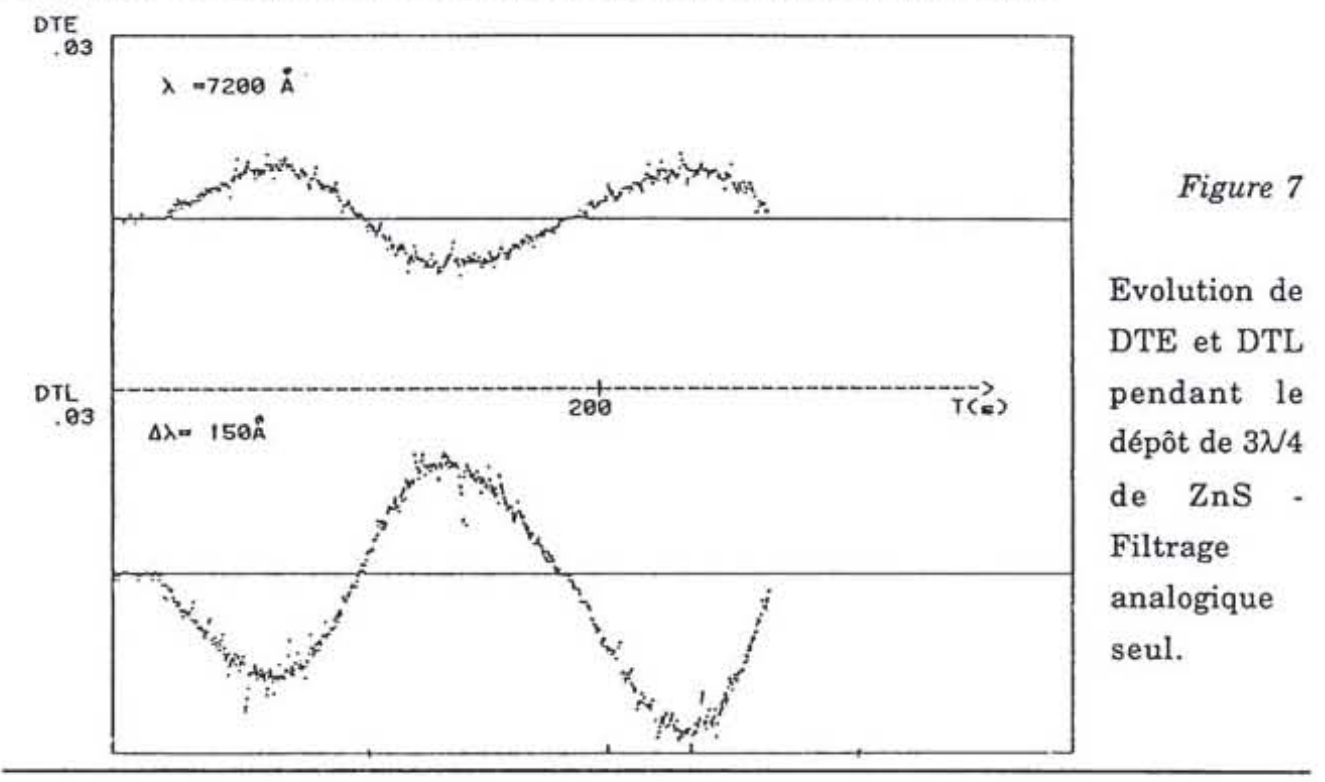

Rappelons pour en terminer avec le contrôle optique d'une couche qu'une mesure faite sur la longueur d'onde $\lambda_{j}$ permet de garantir la réalisation précise d'une couche dont l'épaisseur optique est un multiple entier de $\lambda_{\mathrm{j}} / 4$; la connaissance de la valeur $n\left(\lambda_{j}\right)$ n'est pas nécessaire (avec le repérage du zéro de DTE). Mais l'incertitude sur n amène une erreur sur e. Lorsque le contrôle porte sur le profil $T(\lambda)$, la connaissance de $n(\lambda)$ est indispensable à moins que l'on se contente de copier une expérience précédente. 


\section{V - 4) Généralisation au contrôle d'un empilement}

On veut réaliser le contrôle direct de l'empilement $\mathrm{n}_{\mathrm{S}},\left(\mathrm{n}_{\mathrm{i}}, \mathrm{e}_{\mathrm{i}}\right)_{\mathrm{i}}=1, \mathrm{P}$. Il faut préparer un programme de contrôle. C'est à dire que pour chacune des couches il s'agit de définir le critère d'arrêt retenu : repérage d'extremum ou de zéro de dérivée et longueur d'onde correspondante. La simulation par le calcul de la formation de l'empilement donne le détail de l'évolution attendue.

La Fig. 8 illustre un exemple de formation d'un filtre passe haut. Chacun des profils tracés correspond au profil que l'on doit obtenir à la fin du dépôt de chacune des couches successivement réalisées. Il faut maintenant s'intéresser au détail de l'évolution de chacun de ces profils au voisinage de l'épaisseur requise, pour pouvoir effectuer le choix du

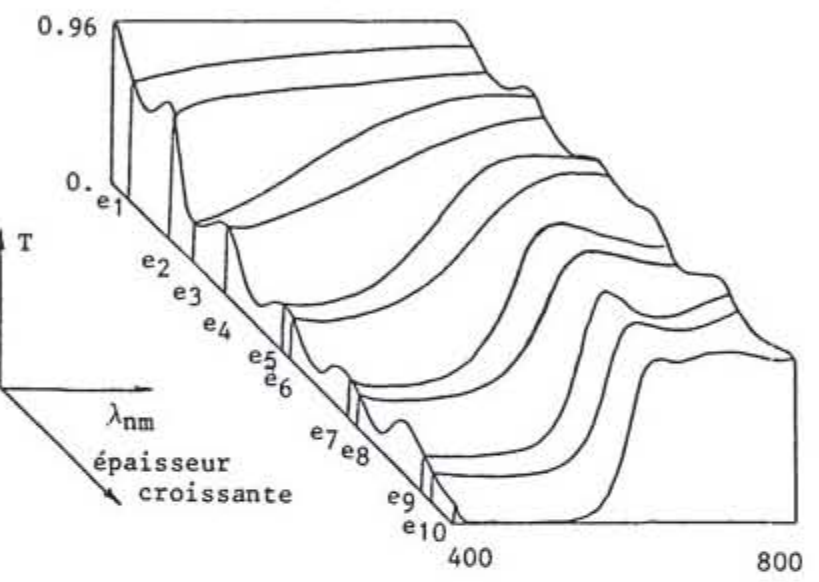
critère d'arrêt que l'on va retenir.

Figure 8

La Fig. 9 concerne le cas de la couche de rang 7 de cet empilement. On y a représenté les profils calculés pour une série d'épaisseurs qui sont respectivement de 0,90 e $7, \mathrm{e}_{7}$, $1,05 \mathrm{e}_{7}$ et $1,10 \mathrm{e}$. On a ainsi une idée de l'évolution du profil lorsque l'on arrive au voisinage de l'épaisseur requise. Le contrôle large bande repose sur une mesure de l'état de coïncidence entre le profil observé au cours du dépôt et le profil théoriquement attendu. Maintenant on peut aussi exploiter le repérage d'extremums ou de zéros des dérivées. On a localisé sur la figure 8 , toutes les longueurs d'onde particulières pour lesquelles une des deux dérivées venait à s'annuler sur le profil correspondant à l'épaisseur e7. On a à priori le choix entre ces diverses longueurs d'onde et il est évidemment préférable de sélectionner celle qui est susceptible de donner la meilleure sensibilité. 


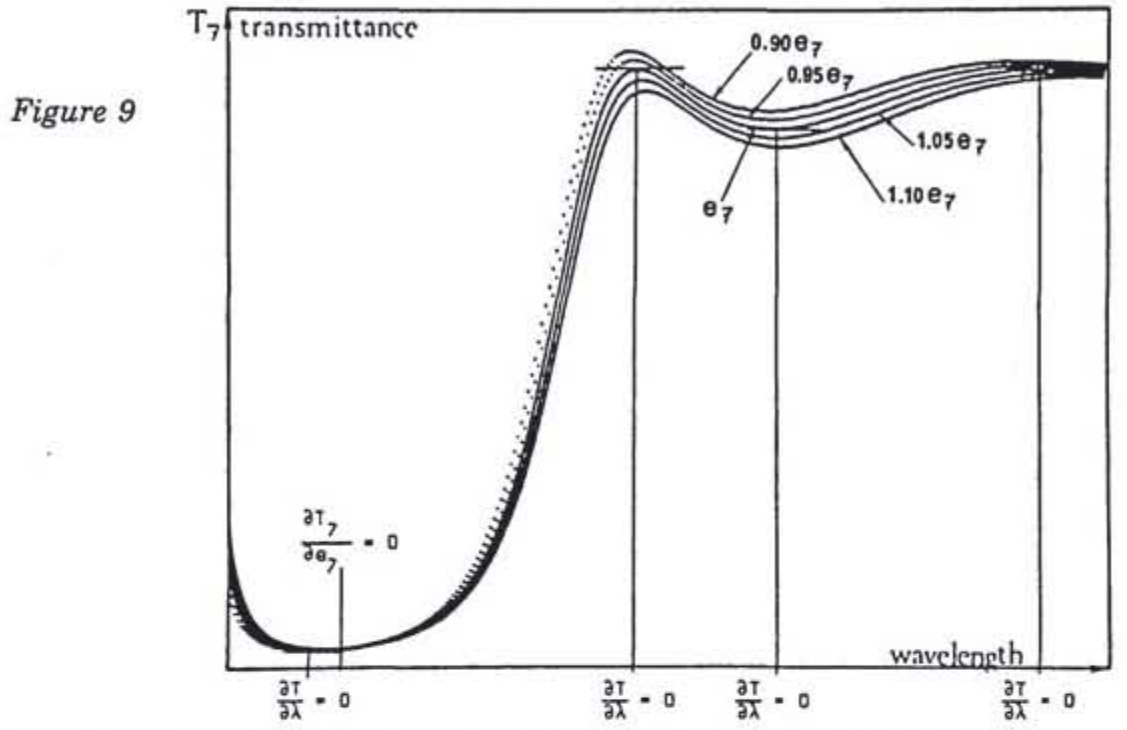

Le calcul, pour le critère d'arrêt choisi, de la sensibilité ainsi que du détail de l'évolution attendue au cours de la formation de la couche (l'épaisseur passe de 0 à e e) ne pose pas de difficultés particulières.

En effectuant ce travail couche par couche, on élabore ainsi tous les éléments qui constituent le PROGRAMME DE CONTROLE de la totalité de l'empilement avec une liste de longueurs d'onde $\Lambda_{1}, \Lambda_{2}, \ldots . \Lambda_{\mathrm{p}}$.

Au cours du dépôt, il reste à suivre scrupuleusement l'ensemble de ces instructions pour garantir la réussite du projet. Mais avant d'en arriver à s'intéresser à l'étape de la réalisation, on va examiner le cas particulier des empilements constitués de couches quart d'onde pour élaborer le programme de contrôle de ce type d'empilement.

\section{V - 4 - 1) Cas des empilements quart d'onde}

Les couches ont toutes une épaisseur optique égale ou multiple de $\lambda_{0} / 4$. On a vu ci-dessus que pour le contrôle de la première couche il suffit d'utiliser la longueur d'onde $\lambda_{0}$ avec la dérivée DTE. : $\Lambda_{1}=\lambda_{0}$. On peut montrer que pour le contrôle de la couche de rang 2 ainsi que pour toutes les suivantes jusqu'à $P, \lambda_{0}$ est une solution particulière parmi les longueurs d'onde possibles pour le contrôle.

On est évidemment tenté de choisir $\Lambda_{1}=\Lambda_{2}=\Lambda_{p}=\lambda_{0}$ parce que l'on ne peut pas rêver d'un programme de contrôle plus simple. 
Si on dispose d'une source monochromatique bien adaptée, il suffit de repérer les extremums de transmission (Fig. 10).

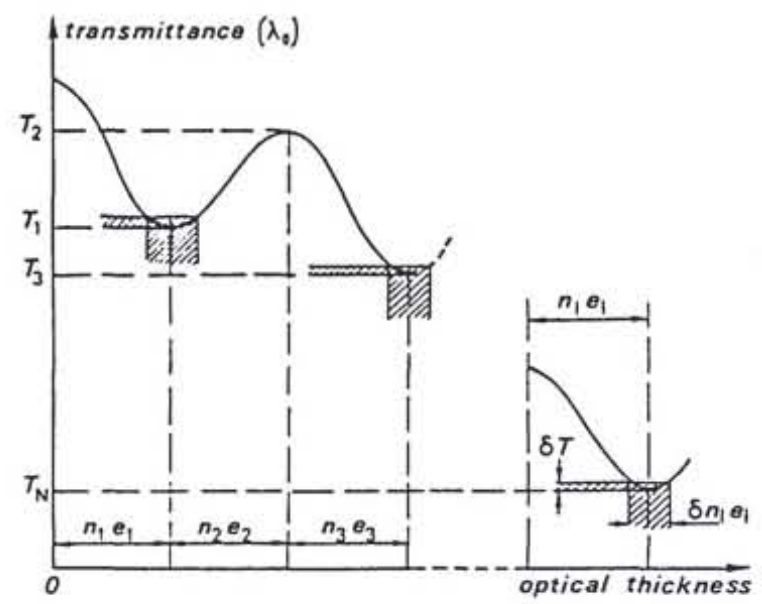

Figure 10

Insistons encore une fois sur le fait que pour un empilement quart d'onde pour $\lambda_{0}$, le calcul du programme de contrôle est apparemment inutile, la longueur d'onde de contrôle étant $\lambda_{0}$. On a ainsi tendance à confondre l'épaisseur optique des couches (c'est à dire la longueur d'onde pour laquelle les couches sont quart d'onde) et la longueur d'onde qu'il faut utiliser pour effectuer le contrôle !

\section{$V$ - 4 - 2) Conséquence des imperfections de contrôle et de réalisation de} multicouches.

Les calculs de simulation permettent d'aborder de façon très générale ce problème qui a une importance cruciale pour les fabricants de couches minces.

La réalisation va s'écarter du projet si :

- les critères d'arrêt ne sont pas exactement respectés. Par exemple pour DTE on arrêtera le dépôt non pas au zéro de DTE mais pour $\mathrm{DTE}_{1}=\varepsilon_{1}$. Les causes d'erreurs sont nombreuses : sensibilité insuffisante, bruit....

- les valeurs effectives des indices de réfraction sous vide ne sont pas connues avec précision. On désignera par le symbole $\mathrm{N}_{\mathrm{i}}$ ces valeurs d'indices.

Partant d'une distribution d'erreurs à priori connues $\left(\varepsilon_{\mathrm{i}}, \mathrm{N}_{\mathrm{j}}\right)$ il est possible de générer l'empilement qui doit effectivement être obtenu.

Le calcul se fait par étapes qui s'enchaînent automatiquement. 
Il s'agit tout d'abord de calculer l'épaisseur $\mathrm{E}_{1}$ de la première couche déposée : $\left(n_{s}, N_{1}, E_{1}\right)$ correspondant à l'équation $D_{T E}-\varepsilon_{1}=0$ pour la longueur d'onde $\Lambda_{1}$.

Pour calculer $\mathrm{E}_{2}$ il faut considérer le système de couches avec les paramètres : $\left(\mathrm{n}_{\mathrm{s}}, \mathrm{N}_{1}, \mathrm{E}_{1}, \mathrm{~N}_{2}, \mathrm{E}_{2}\right)$ où $\mathrm{E}_{2}$ est la variable est fixée par la condition $\mathrm{DTE}_{2}-\varepsilon_{2}=0$ pour $\Lambda_{2}$.

A la fin de l'empilement on a obtenu $\mathrm{n}_{\mathrm{s}}, \mathrm{N}_{1}, \mathrm{E}_{1}, \mathrm{~N}_{2}, \mathrm{~N}_{3}, \mathrm{E}_{3} \ldots \mathrm{N}_{\mathrm{p}}, \mathrm{E}_{\mathrm{p}}$

Il faut bien comprendre que le contrôle de la couche de rang $\mathrm{j}$ ne conduit à l'épaisseur prévue $e_{j}\left(E_{j}=e_{j}\right)$ que si les critères d'arrêt sont rigoureusement respectés et que si les indices $N_{1} \ldots . . N_{j}$ sont identiques aux valeurs théoriques utilisées au départ $\left(\mathrm{n}_{1}, \mathrm{n}_{2} \ldots . . \mathrm{n}_{\mathrm{j}}\right)$.

Si les propriétés optiques de l'empilements "réalisé" $\mathrm{T}\left(\mathrm{N}_{\mathrm{i}} \mathrm{E}_{\mathrm{i}}\right)$ ne différent pas sensiblement des valeurs "attendues" $T\left(n_{i}, e_{i}\right)$ pour le domaine spectral intéressant, le succès est garanti !".

Mais l'effet cumulatif des erreurs peut s'avérer désastreux, au point même que l'on risque de perdre le contrôle.

Ceci nous amène à considérer deux facteurs importants :

- la sensibilité du contrôle; disons qu'en pratique elle est liée à la vitesse de variation du signal en fonction de l'épaisseur.

- la stabilité qui correspond à la manière dont les erreurs peuvent s'accumuler.

L'importance pour la fabrication des filtres est énorme. Ces problèmes ont été largement étudiés par deux équipes dans le monde (Newcastle (Macleod) et à Marseille), il est intéressant de présenter quelques conclusions par l'examen de cas particuliers d'applications.

\section{$V$-4 - 3) Simulation du contrôle d'empilements quart d'onde}

Il est commode de montrer quelques résultats où l'on fait volontairement abstraction du critère de sensibilité pour mieux aborder le problème des erreurs cumulatives. Au lieu d'une erreur sur le critère d'arrêt, on va simplement introduire une perturbation sur la valeur de l'épaisseur avec un $\delta$ ne sous forme d'une fraction de l'épaisseur requise $\delta$ ne (par exemple $\delta$ ne $=0,1$ ne, soit $10 \%$ d'erreur).

Les méthodes de simulation nous permettent de comprendre le mécanisme de compensation des erreurs.

Avec DTE: une erreur sur la couche $j$ perturbe la couche $j+1$ et elle seule,

Avec DTL: une erreur sur la couche $\mathrm{j}$ perturbe toutes les couches suivantes, même si les matériaux ne présentent pas de dispersion.

Note: A cause de la dispersion des matériaux, on ne réalise pas avec un contrôle DTL pour $\lambda_{0}$ un empilement de couches toutes strictement égales à $\lambda_{0} / 4$. 
On résume de façon typique le comportement des contrôles DTE et DTL du point de vue de l'effet cumulatif des erreurs par rapport au cas d'erreurs indépendantes, grâce à la Fig. 11, qui montre les performances optiques des filtres respectivement réalisés avec ces modes de contrôle. Ceci montre clairement que les tolérances de réalisation sont bien supérieures si on utilise DTE. En dépit des erreurs de contrôle, les profils du filtre parfait et du filtre obtenu avec DTE sont identiques. Pour la même distribution d'erreurs, avec le contrôle DTL, on bénéficie d'une compensation d'erreurs qui est moins bonne. Tout est relatif, car dans le cas d'erreurs indépendantes, en gardant toujours la même distribution d'erreurs, le profil du filtre se dégrade très vite.

\section{Figure 11}

Exemple de fabrication avec des erreurs.

Filtre parfait et filtre produit en utilisant DTE avec des erreurs de $10 \%$.

- - - - Filtre produit en utilisant DTL avec des erreurs de $10 \%$.

Erreurs de $10 \%$, cas d'erreurs indépendantes.

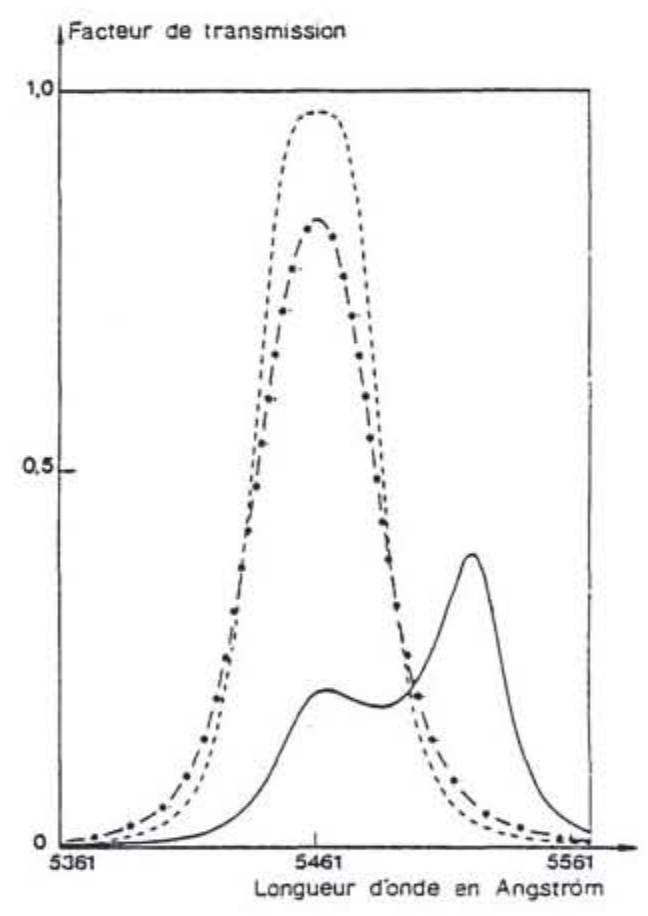

En toute rigueur, il faut tempérer notre conclusion sur la comparaison entre DTE et DTL car la sensibilité de cette dernière est souvent bien supérieure et l'hypothèse d'une confrontation à partir d'erreurs identiques ne plaide évidemment pas en faveur de DTL. 


\section{V - 5) Conclusion sur les techniques de contrôle}

On a introduit ici la notion d'un contrôle stable. Cela met conjointement en jeu :

- la formule de l'empilement

- le procédé de contrôle

- les propriétés optiques intéressantes.

En dépit d'erreurs de réalisation, on peut obtenir malgré tout des propriétés optiques conformes aux tolérances.

Le cas d'un empilement des couches quart d'onde en particulier pour les filtres Fabry-Perot, double onde,.... est criant. L'avantage d'un contrôle optique direct est indéniable; lui seul permet la réalisation des systèmes les plus complexes c'est à dire des filtres à cavités multiples.

La Fig. 12 montre l'évolution qui est observable lors de la formation d'un filtre Fabry-Perot; dans ce calcul les couches sont strictement $\lambda_{0} / 4$. Une bonne solution pour le contrôle consiste à utiliser en plus de la visualisation du profil:

- le contrôle DTE avec l'utilisation des zéros de cette dérivée comme critère d'arrêt pour chacune des couches successivement déposées.

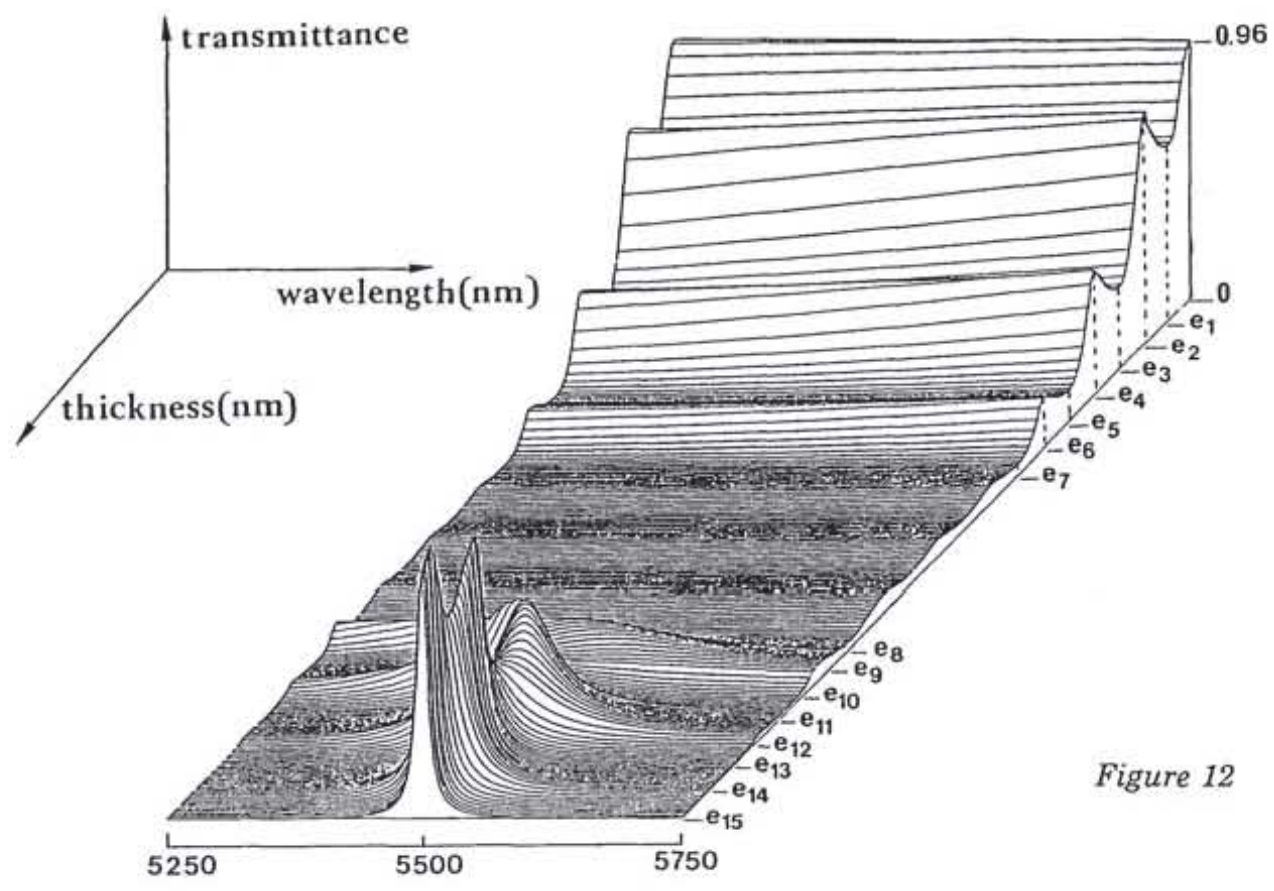


- le contrôle DTL et surtout les valeurs résiduelles de DTL enregistrées à la fin du dépôt de chacune des couches successives. Abstraction faite de la dispersion - dont on peut tenir compte - la coïncidence entre les zéros de DTE et de DTL (le filtre présente une transmission maximale et doit être parfaitement centré) implique que les couches réalisées sont strictement quart d'onde.

DTL est donc un moyen pour vérifier que le critère d'arrêt DTE $=0$ a été bien respecté. Et on a donc ainsi UN CONTROLE DU CONTROLE!

Dans le cas général d'empilements de couches d'épaisseurs optiques inégales et même dans le cas d'empilements quart d'onde où les propriétés optiques intéressantes ne concernent pas uniquement un étroit domaine autour de la longueur d'onde de centrage, les choses sont loin d'être aussi simples. Il n'y a plus de longueur d'onde particulière $\lambda_{0}$; dès lors les méthodes de contrôle à large bande sont à priori préférables. Elles ont en tout cas l'avantage d'éviter les fastidieux changements de longueur de contrôle lorsque l'on passe d'une couche à l'autre lors de la formation de l'empilement.

Une solution à priori évidente consiste à exploiter les profils spectraux mesurés en temps réel. En s'appuyant sur une fonction de mérite qui représente la distance entre le profil mesuré et le profil attendu, on peut forger un critère d'arrêt qui correspond à l'annulation de cette distance. Cette méthode n'est pas sans inconvénient. Elle implique d'abord une parfaite connaissance des indices de réfraction des couches dans les conditions mêmes de production, enfin elle suppose que l'on est capable d'assurer une prédiction correcte des profils qui doivent successivement être obtenus en passant d'une couche à l'autre dans la construction de l'empilement. Les résultats expérimentaux sont souvent décevants dès qu'il s'agit d'un empilement avec un nombre élevé de couches. La théorie diffère de l'expérience, et ceci tient à de multiples effets. On ne maîtrise pas suffisamment les épaisseurs des couches individuelles, la répétabilité des indices n'est pas totalement garantie; plus grave encore on s'aperçoit que les calculs des propriétés optiques ne sont pas forcément fiables et ceci nous amène à des études spécifiques pour mieux appréhender la microstructure des coucheș minces et le comportement effectif que celles-ci sont susceptibles d'avoir.....

Quelques remarques complémentaires s'imposent avant de conclure sur le chapitre concernant les méthodes de contrôle.

On a beaucoup examiné le contrôle avec des mesures de transmission. Que peuton dire des mesures de réflexion? Le tableau suivant résume la situation concernant les mesures de $\mathrm{T}$ ou de $\mathrm{R}$ pour un contrôle optique. 


\begin{tabular}{|c|c|c|}
\hline & Fact. de transmission & Facteur de réflexion \\
\hline Calibrage & - Calibrage du zéro & - Calibrage du zéro \\
\hline à & - Calibrage de 0,96 = T & - Calibrage de $0,04=R$ \\
\hline réaliser & du substrat nu & du substrat nu \\
\hline Inconvénients & & $\begin{array}{l}\text { - Trop sensible au } \\
\text { mouvement de rotation } \\
\text { du substrat }\end{array}$ \\
\hline Avantages & $\begin{array}{l}\text { Pas de difficulté avec } \\
\text { l'incidence normale. } \\
\text { Bien adapté pour les } \\
\text { miroirs et les filtres. }\end{array}$ & $\begin{array}{l}\text { Très intéressant pour } \\
\text { les antireflets }\end{array}$ \\
\hline
\end{tabular}

$\rightarrow \quad$ Il faut revenir sur l'option à prendre : contrôle direct ? ou contrôle indirect ? ou la solution intermédiaire d'un contrôle semi-direct?

En pratique, chaque empilement est un cas particulier et l'on doit l'étudier en tant que tel. En général on préferera un contrôle direct si on bénéficie d'une sensibilité suffisante. Dans quelques cas, un prétraitement s'avère très utile: par exemple un témoin couvert d'un couple de couches B H va donner une bien meilleure sensibilité et une stabilité remarquable. Cette technique est très utile pour le contrôle de nombreux antireflets utilisés dans l'industrie.

Les techniques de simulation peuvent nous aider dans le choix du mode de contrôle: en effet, on peut théoriquement réunir toutes les informations dont on a besoin pour une estimation des tolérances de nos filtres.

Rappelons-en les grandes lignes:

- la synthèse nous a donné un empilement de couches

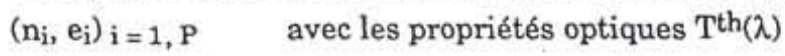

- la répétabilité de la production des indices est donnée par une distribution gaussienne, les fluctuations sont $\delta n_{i}$

- le programme de contrôle de l'empilement étant défini, on doit partir de la connaissance de la distribution d'erreurs commises sur les critères d'arrêt pour calculer :

- la sensibilité de la méthode de mesure de l'épaisseur $\mathrm{e}_{\mathrm{i}}$, l'erreur est $\delta \mathrm{e}_{\mathrm{j}}$

- et les conséquences par effet cumulatif au niveau du contrôle des couches suivantes. 
On aboutit à tout un ensemble de solutions du type :

$\left(N_{i}, E_{i}\right)_{i}=1, P$ avec des propriétés optiques $T^{e f f}(\lambda)$

La distance $\quad\left\|T^{\text {th }}(\lambda)-T^{\text {eff }}(\lambda)\right\| \quad$ rend directement compte de la qualité du point de vue des performances optiques qui intéressent l'utilisateur.

Ainsi dès le niveau de l'étape de synthèse on peut en principe avoir quelques indications sur nos chances d'aboutir à un produit correct.

Pour une production industrielle on est tenté de parler en terme de rendement et on va évidemment chercher à comparer nos pronostics avec une analyse statistique portant sur les résultats effectivement obtenus. La confrontation de l'expérience avec le calcul n'est malheureusement pas toujours aussi simple que l'on pourrait le souhaiter. Ces questions font l'objet du chapitre suivant où l'on va surtout s'intéresser aux aspects expérimentaux de la physique des couches minces.

\section{VI - COUCHES MINCES: LIMTTATIONS DUES A LA MICROSTRUCTURE}

La plupart des difficultés de réalisation s'expliquent par le fait que les couches ont, sous vide, un comportement différent du "comportement mathématique" que l'on est en droit d'attendre.

- Comment contrôler les propriétés effectives des couches?

- Comment contrôler le procédé de contrôle et l'empêcher de diverger?

\section{VI - 1) Stoechiométrie}

Au cours du dépôt sous vide, la plupart des matériaux ont des propriétés optiques qui ne sont pas conformes aux valeurs attendues. Il faut admettre que la stoechiométrie du matériau condensé ne correspond pas à celle désirée.

Exemple: $\mathrm{TiO}_{2}$

La Fig. 13 donne une

idée des propriétés mesurées sous vide et à l'air si la composition n'est pas "maîtrisée en temps réel". Les conditions de dépôt doivent être réglées pour éliminer ces problèmes.

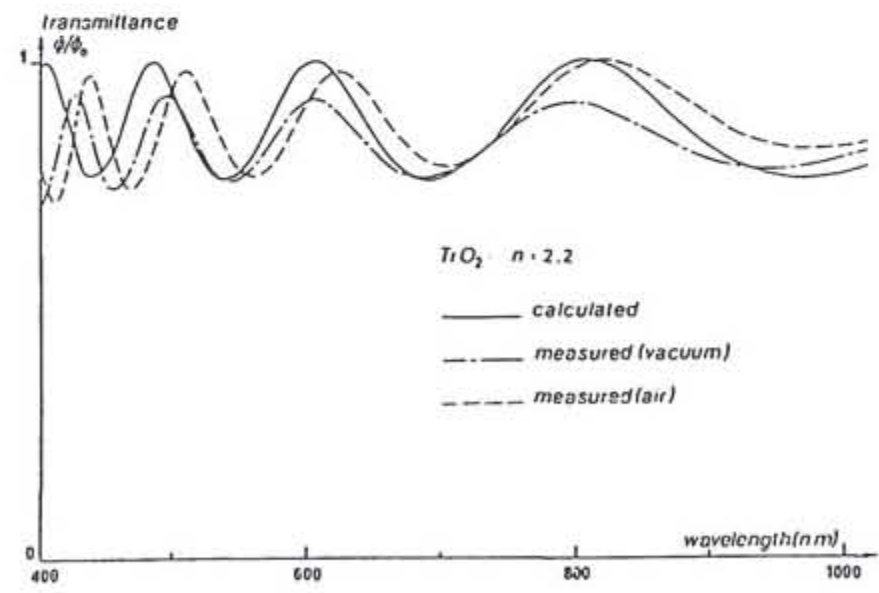




\section{VI - 2) Comment contrôler le contrôle?}

On peut exploiter les mesures faites in situ. Si on rajoute une couche sur un empilement, on va enregistrer pour une longueur d'onde un signal comme celui donné Fig. 14. La connaissance des caractéristiques de l'empilement n'est pas nécessaire pour déterminer $\mathrm{n}$ et $\mathrm{d}$ de la couche. Cette détermination se fait à partir des quatre valeurs mesurées $\mathrm{T}_{\mathrm{j}-1}, \mathrm{~T}_{\mathrm{M}}, \mathrm{T}_{\mathrm{m}}, \mathrm{T}_{\mathrm{j}}$. Le travail peut être effectué simultanément pour une série de longueurs d'onde. Il reste à éviter par tous les moyens des couches trop inhomogènes!

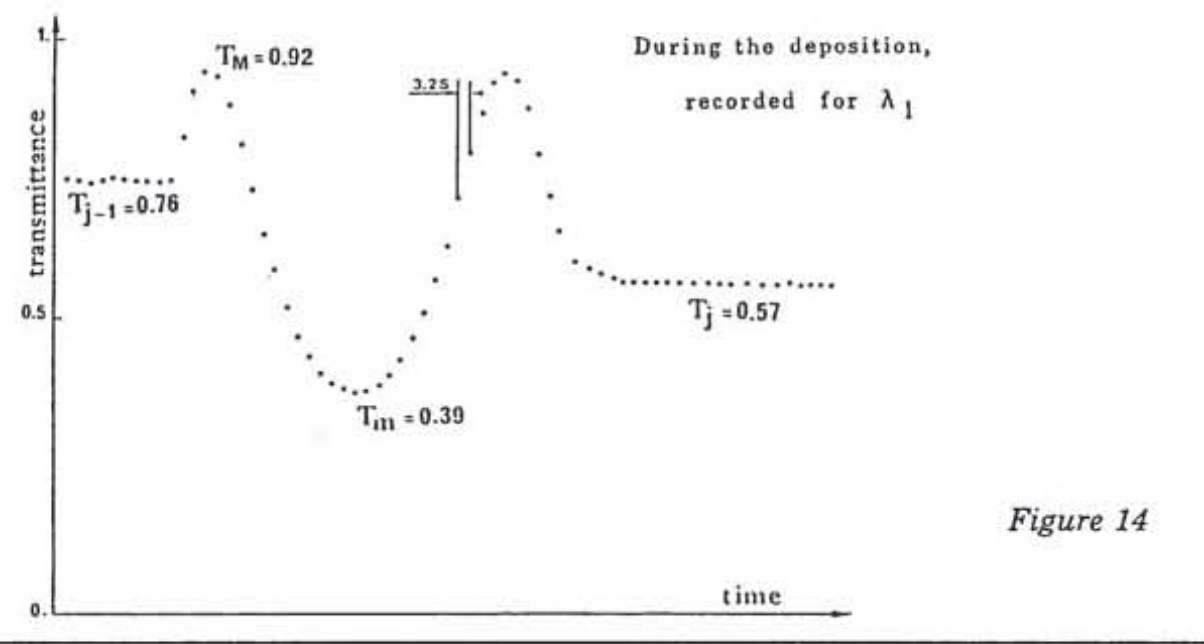

\section{VI - 3) Effet cumulatif des erreurs de réalisation}

On a étudié la compensation possible dans le cas d'erreurs statiques.

Par le terme "erreur statique" il faut comprendre: une erreur de réalisation; la couche n'est pas exactement conforme à la valeur désirée et on suppose implicitement que cette erreur n'évolue pas au cours du temps.

Que se passe-t-il si les erreurs évoluent, c'est-à-dire si, au cours du dépôt, les propriétés optiques $\left(\mathrm{n}_{\mathrm{i}} \mathrm{e}_{\mathrm{j}}\right)$ de l'empilement déjà réalisé évoluent?

On dit que l'on a des erreurs dynamiques. Les causes possibles sont:

- Oxydation non terminée à la fin du dépôt d'une couche;

- Variation de température qui entraîne:

- dilatation sur les valeurs d'indice

- dilatation de l'épaisseur; 
- Adsorption spontanée de l'humidité résiduelle dans l'enceinte à vide.

La dépendance de l'uniformité avec le temps (émission des creusets avec une indicatrice qui varie au cours du temps) interdit le traitement simultané de nombreux filtres si les tolérances de réalisation (au sens des erreurs indépendantes) sont sévères.

\section{VI - 4) Indices in situ et indices à l'air-Méthodes de détermination}

1) in situ (méthode décrite ci-dessus)

2) A l'air. On mesure les propriétés optiques $R(\lambda)$ et $T(\lambda)$ d'une monocouche. La détermination des constantes opto-géométriques est un problème de synthèse que l'on résout par itérations. On part souvent de l'hypothèse d'une couche inhomogène à gradient constant d'indice (Fig. 15). A partir de $\mathrm{R}(\lambda)$ et $\mathrm{T}(\lambda)$, on extrait: $\hat{\mathrm{n}}(\lambda),(\Delta \mathrm{n} / \mathrm{n})_{(\lambda)}, \mathrm{e}, \mathrm{k}(\lambda)$. La Fig. 16 donne un exemple de résultat avec lincertitude sur n (barres d'erreur).

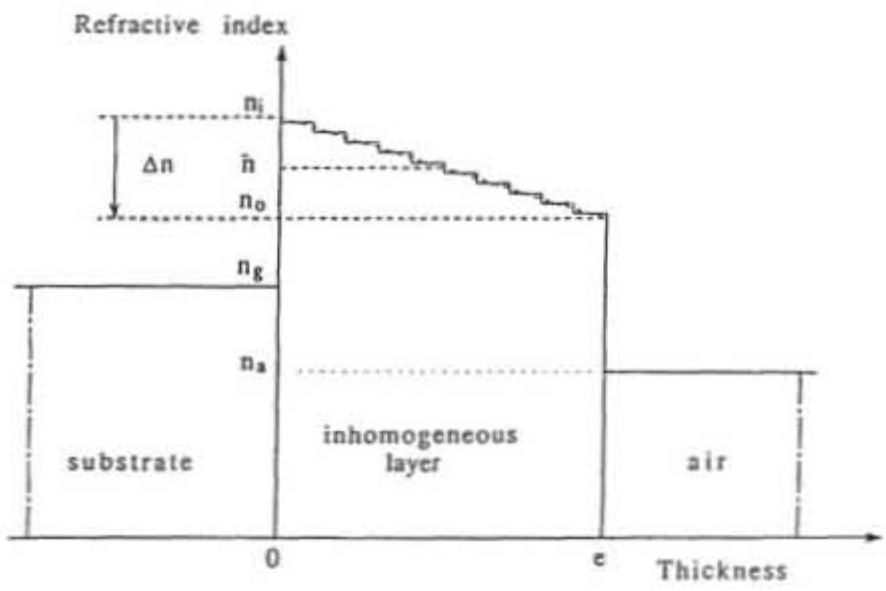

Figure 15

Figure 16

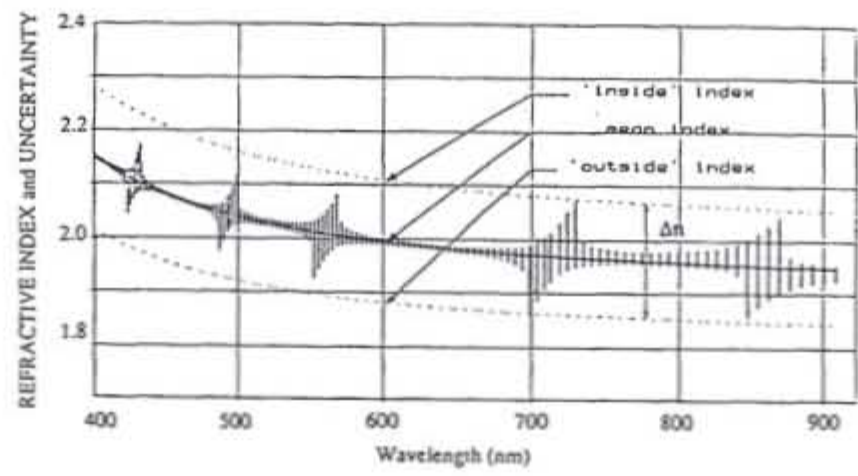


La détermination de l'inhomogénéité $\Delta \mathrm{n} / \tilde{\mathrm{n}}$ repose sur les mesures pour les longueurs d'onde particulières pour lesquelles la couche a une épaisseur optique multiple de $\lambda / 2$ (pour ces longueurs d'onde une couche homogène serait "absente").

La Fig. 17 (a, b, c, d) donne les résultats pour $\mathrm{TiO}_{2}$ et $\mathrm{SiO}_{2}$ sous vide et à l'air pour différentes conditions de dépôt.
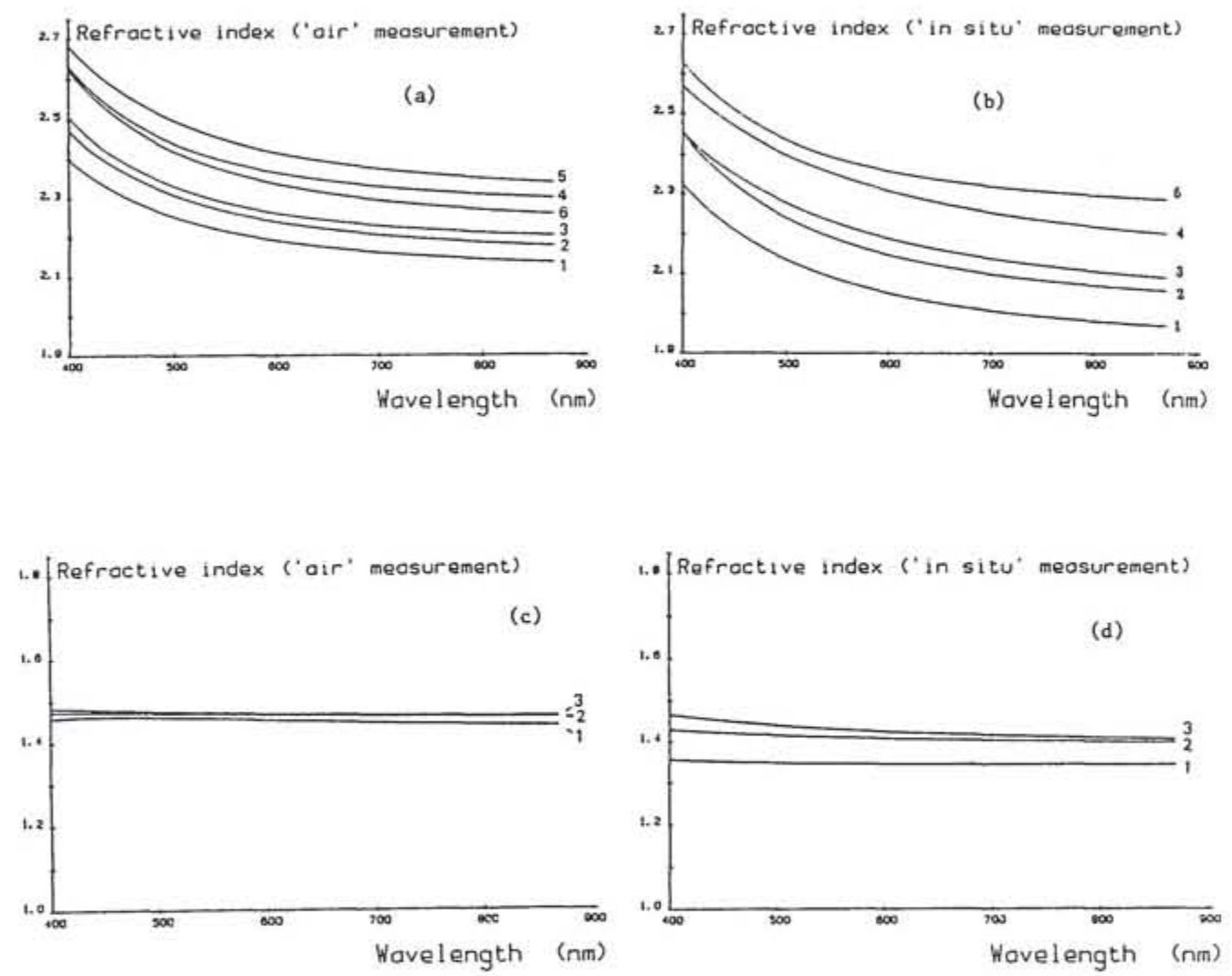

Figure $17(a, b, c, d)$

\section{Interprétation des différences entre vide et air}

En premier lieu, on a un effet qui est lié à la variation de température. Il faut considérer un coefficient de dilatation de l'indice et un coefficient de dilatation de l'épaisseur. En fait, on aura souvent accès à une mesure du produit (ne) $)_{\theta}=n_{0}(1+\gamma \theta)$ où $\gamma$ est le coefficient de dilatation de la couche (par exemple de l'ordre de $510^{-4}$ pour le sulfure de zinc; bien plus faible pour le $\mathrm{TiO}_{2}$ ). 
En réalité, le principal effet pour expliquer la différence entre vide et air est lié à la pénétration d'eau dans les couches par adsorption spontanée.

A ce sujet, les tentatives de modélisation sont vouées à l'échec sauf dans de rares cas particuliers.

\section{VII - TECHNIQUES DE MESURE DES PERTES ET D'ETUDE DES DEFAUTS DE STRUCTURE DANS LES COUCHES}

Jusqu'à présent, nous nous sommes intéressés aux mesures des facteurs de réflexion et de transmission et on a surtout présenté l'ensemble des moyens théoriques et expérimentaux qui ont été forgés pour permettre la réalisation de traitements de surface ayant des propriétés spectrales déterminées. On a vu que les couches étaient souvent loin d'avoir des propriétés optiques idéales, c'est-à-dire strictement conformes aux prévisions calculées. Nous avons introduit dans les calculs l'absorption en ayant un indice de réfraction complexe $\widetilde{\mathrm{h}}=\mathrm{n}$ - ik où le coefficient d'extinction $\mathrm{k}\left(\mathrm{k} \approx 10^{-5}\right.$ dans les meilleurs cas) est bien plus important que celui mesuré sur les matériaux massifs correspondants (de l'ordre de $10^{-11}$ pour la silice!).

Le modèle simple de couche n'étant souvant pas suffisant pour rendre compte des performances effectives, on a également introduit une notion de couche inhomogène en s'intéressant particulièrement à la détermination du gradient d'indice dans une direction perpendiculaire aux faces. On va maintenant s'intéresser en détail aux techniques de mesure qui nous permettent d'aller plus loin dans la compréhension des facteurs qui viennent limiter les performances des empilements. Notre objectif reste toujours le même puisqu'il s'agit de la caractérisation et du développement de modèles de couches pour permettre une prédiction aussi correcte que possible des empilements que l'on cherche à fabriquer. Ceci nous amène naturellement à étudier le mécanisme des pertes dans les couches avec une introduction du phénomène de la diffusion dans un bilan énergétique complet.

\section{VII - 1) Pertes par diffusion}

A cause des rugosités des surfaces et interfaces d'un empilement ainsi que des hétérogénéités de volume, une partie de la lumière se trouve diffractée (ou plus exactement diffusée) en dehors des directions spécualires de réflexion et de transmission. Dans ces conditions, le bilan d'énergie doit comprendre un terme complémentaire $\mathrm{D}_{\mathrm{G}}$ qui représente la quantité globale de lumière diffusée dans l'espace autour du point éclairé: 


$$
\mathrm{R}+\mathrm{T}+\mathrm{A}+\mathrm{D}_{\mathrm{G}}=1
$$

Les composants multicouches que l'on sait fabriquer aujourd'hui présentent des pertes globales $\mathrm{A}+\mathrm{D}_{\mathrm{G}}$ qui n'excèdent pas quelques millièmes et il nous faut des techniques extrêmement précises pour étudier chacunb des deux termes.

En ce qui concerne $\mathrm{D}_{\mathrm{G}}$, on peut évidemment collecter point par point dans tout l'espace le flux de lumière diffusée par un échantillon éclairé dans des conditions bien déterminées. Quelques appareillages existent dans le monde; il faut imaginer un système mécanique assez complexe où un récepteur se déplace à volonté sur une sphère entourant l'échantillon. Encore une fois, les problèmes de calibration sont extrêmement importants et il s'agit de mesurer ici des flux très faibles puisque dans certaines directions de l'espace l'intensité diffusée peut être bien inférieure au millionième du flux pris dans une des deux directions spéculaires de réflexion ou de transmission. On utilise comme unité le BRDF (Bidirectional Reflectance Distribution Function) qui représente l'intensité du flux par unité d'angle solide (stéradian). Il est commode de signaler l'analogie avec la notion de probabilité pour la répartition de la quantité de lumière diffusée dans chacune des différentes directions de l'espace. En effectuant la somme de toutes ces mesures, on accède à la valeur de la diffusion globale. Maintenant, si on tient compte des flux réfléchis et transmis dans les directions spéculaires, la somme des "probabilités de présence du photon" est égale à l'unité pour respecter la loi de conservation de l'énergie (avec un terme de correction pour l'absorption).

En dépit de quelques difficultés instrumentales pour couvrir avec une densité d'échantillonnage suffisante la totalité de l'espace, on accède à une mesure de l'indicatrice de diffusion (BRDF en fonction des coordonnées de l'espace $\theta$ et $\varphi$ ) et à la quantité globale de lumière diffusée.

La précision est excellente; par exemple, pour un miroir de cavité laser, on a tracé sur la Fig. 18 la section dans le plan d'incidence de l'indicatrice mesurée. Pour ce miroir, la diffusion globale est de $3.10^{-4}$ avec une incertitude ne dépassant pas quelques $10^{-5}$.

Figure 18

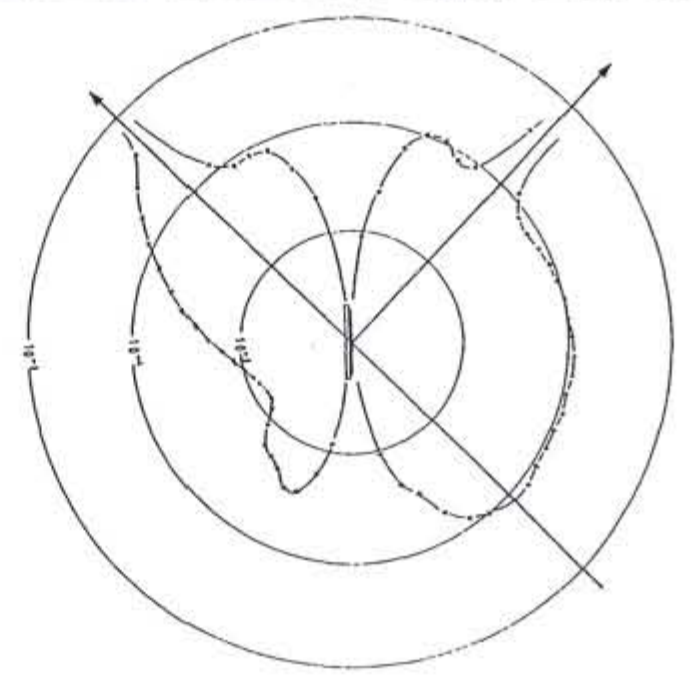


Pour les composants optiques de qualité, le mécanisme des pertes par diffusion est bien compris et relativement bien maîtrisé. De nombreux auteurs s'accordent [5], [6] pour montrer que la diffusion est due pour l'essentiel aux rugosités de la surface du substrat et des interfaces de l'empilement. En comparaison, avec la plupart des matériaux utilisés, les effets dus aux hétérogénéités d'indice dans le volume des couches étant bien plus faibles. La théorie montre que c'est le coefficient de corrélation entre les interfaces qui est un paramètre clé de la diffusion.

Malgré l'excellence de tous ces résultats de caractérisation, on veut encore aller plus loin et ceci nous amène d'abord à une étude de la diffusion sur des couches isolées. L'idée est simple: si on arrive à déterminer l'ensemble des paramètres pour rendre compte du phénomène de diffusion, on devrait assurer l'extrapolation pour la prédiction des pertes d'un multicouche.

Ceci suppose évidemment la connaissance de la loi de composition des défauts entre interfaces successives. Ces travaux donnent alors à l'expérimentateur les solutions théoriques d'empilements les mieux adaptés à son problème particulier: choix des matériaux, des épaisseurs des couches, de la technique de dépôt, .... pour un substrat ayant une qualité bien adaptée.

A titre d'exemple, on a fait appel à ces techniques pour le développement de filtres composant des démultiplexeurs pour les télécommunications optiques. Dans ce problème, on doit faire intervenir pour chacune des longueurs d'onde mises en jeu la répartition spatiale de lumière diffusée par les différents composants. On peut alors chercher à ajuster la position géométrique des différents composants pour éliminer au mieux toute lumière spectrale parasite et réduire la diaphonie.

Pour fixer les idées, il faut retenir que les mesures de diffusion sur un substrat nu (qui ne diffuse pas par le volume, ni par la face arrière) peuvent donner pour le domaine de fréquences spatiales qui nous intéresse une mesure de la fonction d'autocorrélation de la surface et on accède à la mesure de la rugosité même si celle-ci est de l'ordre de $1 \AA$. Avec des traitements multicouches classiques, les rugosités des interfaces sont de l'ordre de 5 à $10 \AA$; ceci dépend toutefois beaucoup des matériaux et des techniques de dépôt utilisées.

\section{VII - 2) Pertes par absorption}

Rappelons que même avec les meilleurs spectrophotomètres, il est bien difficile d'effectuer des mesures des facteurs de réflexion et de transmission avec une précision 
meilleure que 2 ou 3 millièmes. Il faut des techniques plus précises que la spectrophotométrie pour étudier l'absorption.

Plusieurs solutions ont été proposées. Elles utilisent en particulier la microcalorimétrie, la photoacoustique et surtout la déflexion photothermique [7]. La Fig. 19 rappelle le principe de cette dernière méthode.

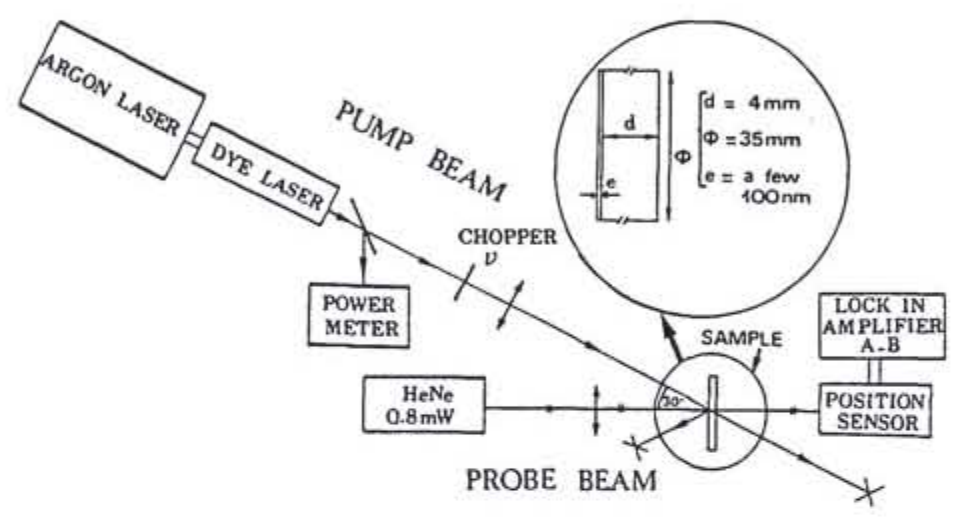

Figure 19

A l'endroit où l'échantillon est éclairé par un faisceau pompe, il se produit un échauffement proportionnel au taux d'absorption A. On peut détecter la gradient de température au voisinage de ce point grâce à un faisceau sonde qui va se trouver défléchi par les variations locales d'indice liées à ce gradient. Une cellule à deux ou quatre quadrants permet de détecter sans difficulté un "effet mirage" de quelques 10-8 radian. Pratiquement on décèle ainsi des pertes par absorption de l'ordre de $10^{-6}$. Sur une couche isolée, le seuil de détection sur $\mathrm{k}$ est de l'ordre de $10^{-7}$, ce qui est tout-à-fait suffisant pour des études de couches minces. La calibration de cet appareillage pose évidemment quelques difficultés qui semblent bien résolues par recoupements entre différentes mesures.

\section{VII - 3) Etude des anomalies de structure}

Nous avons vu plus haut comment on arrivait à mettre en évidence des insuffisances notoires du modèle de couche parfaite dès qu'il s'agissait d'interpréter des résultats de mesure.

Ainsi avec la spectrophotométrie classique, on a pu introduire la notion d'inhomogénéité d'indice et même accéder à une mesure du gradient $\partial \mathrm{n} / \partial \mathrm{z}$. Les mesures d'indicatrice de diffusion nous amènent à la caractérisation des rugosités de surfaces et d'interfaces. On peut évidemment envisager les techniques de mesures 
optiques les plus diverses pour tenter de compléter cette étude des anomalies de structure. On doit disposer de techniques de caractérisation et ceci implique l'emploi de modèles choisis pour leur large domaine de validité. Dès lors, la question la plus difficile à résoudre concerne justement les moyens qu'il s'agit de mettre en œuvre pour l'élaboration de tests suffisants pour s'assurer de la validité de nos modèles!

Les photos de microscopie électronique montrent que les couches qui sont obtenues par une simple évaporation ont une microstructure en forme de colonnes bien analogue à celle que l'on observe avec le basalte. L'orientation des colonnes pour des susbtrats immobiles pendant l'évaporation est directement corrélable avec la direction du flux de matière incident. Ainsi, parce que la compacité est supérieure dans la direction des colonnes que dans les autres directions de l'espace, on doit s'attendre à mettre en évidence une dépendance de l'indice avec la direction de propagation de la lumière. Ceci nécessite des mesures précises.

Pour la détermination de l'indice de réfraction on peut utiliser la technique de mesure des angles de synchronisme avec la détection des lignes noires. Le principe consiste à utiliser un prisme à réflexion totale pour coupler la lumière dans la couche que l'on veut caractériser. Si on éclaire le système dans une direction qui correspond à un mode guidé, et si le gap d'air entre le prisme et l'échantillon est bien adapté, on peut alors transférer de l'énergie dans le guide où cette énergie se propage sur une petite distance. On observe alors dans le faisceau réfléchi une ligne noire qui correspond à un couplage de la lumière. L'angle de synchronisme correspond à la direction pour laquelle on a une ligne noire.

Théoriquement, il suffit de mesurer les angles de synchronisme relatifs à deux modes de propagation; on peut alors écrire deux équations faisant intervenir l'indice $\mathrm{n}$ et l'épaisseur e pour déterminer ainsi ces deux paramètres. Si on dispose de plus de deux modes, il est évidemment tentant d'essayer de détecter un défaut d'homogénéité.

Nous allons montrer comment on peut étudier ainsi l'anisotropie induite par la microstructure. Pour cela on va s'appuyer sur le modèle d'HOROWITZ et MACLEOD schématisé Fig. 20. Les colonnes font un angle a avec la normale au substrat. Le milieu constituant la couche est anisotrope avec trois indices principaux $\mathrm{n}_{1}, \mathrm{n}_{2}, \mathrm{n}_{3}$. La direction des axes principaux est indiquée sur la figure. Il faut considérer une onde guidée qui se propage à l'intérieur de la couche et on se place de telle façon que l'axe de croissance des couches soit situé dans le plan d'incidence du faisceau de mesure. Dans l'hypothèse d'une couche homogène, on conduit les calculs d'indice de la manière suivante: partant des modes TE, on détermine d'abord l'indice $\mathrm{n}_{2}$ et l'épaisseur $\mathrm{d}$ comme dans le cas d'une couche isotrope; 2 modes TE suffisent donc. 
Figure 20:
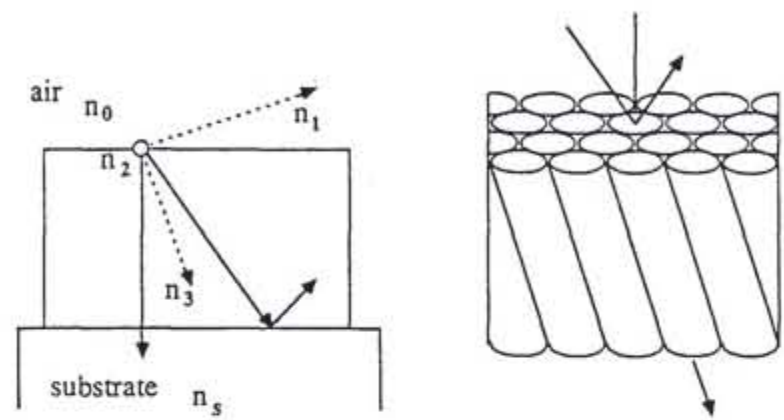

Modèle de structure en colonnes de la couche anisotrope (d'après $F$. HOROWITZ et H.A. MACLEOD)

Une fois $d$ connu, deux modes TM nous conduisent aux valeurs $n_{1}$ et $n_{3}$. On peut évidemment avoir quelques problèmes d'interprétation lorsque l'on dispose de plus de 2 modes TE ou de 2 modes TM et que l'on s'intéresse aux recoupements possibles.

Il n'est pas utile d'aller ici dans tous les détails de cette étude de l'anisotropie car il est facile d'imaginer la complexité des problèmes que l'on va rencontrer avec des multicouches et des conditions d'éclairement hors des plans principaux!

Dans le cas général, les substrats sont animés d'un mouvement de rotation et la formation des colonnes s'en trouve perturbée. L'optique guidée est un moyen précieux pour étudier l'uniformité des dépôts. A titre d'information, on montre ici (Fig. 21) un exemple de résultat concernant l'étude de l'uniformité de l'indice et de l'uniformité d'épaisseur sur un disque plan de $150 \mathrm{~mm}$ de diamètre. Les mesures d'indice sont réalisées pour la longueur d'onde $\lambda=632,8 \mathrm{~nm}$; il s'agit ici d'une couche de $\mathrm{TiO}_{2}$ déposée par la technique IAD et le défaut d'anisotropie est relativement modeste.

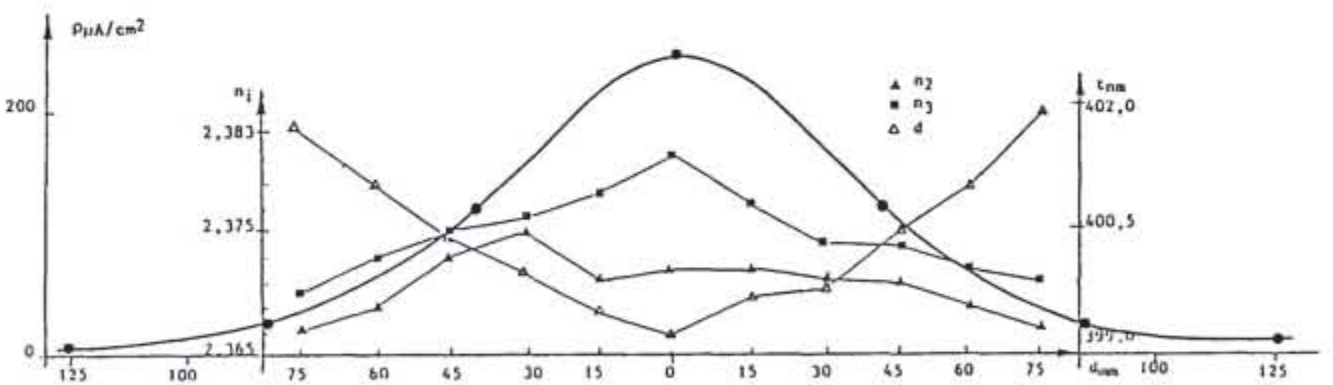

Figure 21 


\section{VII - 4) Conclusion}

Dans la pratique, lorsque l'on veut réaliser un empilement, il est impossible d'introduire tous les termes correctifs pour garantir une prédiction parfaite du système multicouche que l'on veut fabriquer. On maîtrise mal de nombreux paramètres; l'inhomogénéité d'indice, l'absorption, ... Tout ceci complique singulièrement la réalisation de certains empilements.

\section{VIII-CONCLUSIONS ET FUTURS DEVELOPPEMENTS}

- La principale limitation dans l'obtention de filtres ayant des performances poussées tient au fait que les couches n'ont généralement pas un comportement strictement conforme aux prévisions calculées.

Faute de prévisions sérieuses, l'expérience a bien peu de chances d'aboutir facilement à un résultat satisfaisant.

Les méthodes de contrôle optique peuvent nous donner:

- une bonne précision sur l'épaisseur

- et une mesure de l'indice est possible mais le trajet optique dans la couche doit être de l'ordre de $\lambda / 2$.

On retiendra toutefois que les méthodes optiques (qui seules permettent d'accéder à la valeur de l'indice) ne permettent pas de mesurer les variations locales d'indice dans un aggrégat. On ne mesure que la valeur moyenne $\mathrm{n}$ dans une couche d'épaisseur optique $2 / 2$.

\section{VIII - 1) Comment améliorer la production des coatings?}

Deux voies sont à explorer conjointement:

La première vise à améliorer la structure. On a vu que les techniques IAD (Ion Assisted deposition) et IBS (sputtering par faisceau d'ions) semblent être de bonnes voies pour avoir des couches réelles plus proches du modèle de couche parfaite (on élimine ainsi tous les problèmes d'instabilité des indices par adsorption d'eau).

La seconde voie est d'améliorer la connaissance des indices in situ et la répétabilité de l'indice réalisé.

Ceci implique de sérieuses et multiples précautions dans la définition des paramètres de dépôt, du conditionnement des matériaux dans les creusets, du réglage de la pression des gaz résiduels dans l'enceinte, etc ... 
Les calculs de simulation permettent de choisir la méthode de contrôle optique la mieux adaptée. Le système optique de contrôle doit être particulièrement soigné; seules des mesures précises de spectrophotométrie conduisent à un contrôle correct des épaisseurs.

Enfin il est prudent d'assurer une automatisation systématique des opérations: préchauffage, évaporation, ... arrêt, tout doit être piloté par ordinateur pour éviter l'erreur humaine.

\section{VIII - 2) Peut-on corriger en temps réel?}

On doit évidemment envisager l'intérêt potentiel d'une correction en temps réel. Remarquons toutefois qu'il vaut mieux éviter de commettre des erreurs et c'est la raison pour laquelle toutes les opérations doivent être automatisées. En effet, si on élimine toutes les erreurs importantes (avec un système automatique), les seules qui subsistent sont dues à notre incapacité de calculer avec précision. Il faudrait en particulier arriver à prendre en compte l'inhomogénéité. Un processus de correction en temps réel doit s'effectuer en plusieurs étapes qui sont les suivantes:

1) Il faut d'abord détecter l'erreur sur les propriétés optiques dès qu'elle apparaît;

2) On doit arriver à expliquer cette erreur par une erreur de réalisation sur la formule de l'empilement déjà réalisé. L'identification de l'erreur amène à préciser le rang de la couche concernée, la valeur de l'indice obtenu, l'épaisseur déposée et le cas échéant le taux d'inhomogénéité de cette couche.

3) C'est maintenant un calcul de synthèse qui doit nous permettre de choisir les épaisseurs et indices des couches restant à déposer pour avoir le meilleur résultat possible

4) Pour pouvoir assurer la réalisation des épaisseurs calculées en 3) on doit établir un programme de contrôle.

La complexité d'une correction en temps réel par ordinateur est telle que sa mise en œuvre soulève de très sérieuses difficultés.

On n'oubliera pas que dans certains cas tout ce travail est automatiquement assuré par la lumière qui se charge de détecter les erreurs et modifie en conséquence le programme de contrôle 4) des couches suivantes. Dans ce cas, on remplace un ordinateur qui a un travail considérable à faire ... par un mode de contrôle astucieusement choisi. 


\section{VII - 3) Synthèse et tolérances de réalisation}

Dans un cours détaillé on donnerait ici quelques exemples pour montrer comment on doit arriver à prendre en compte au niveau de la synthèse de l'empilement le problème des tolérances de réalisation.

La formule à retenir doit être d'une réalisation facile. On n'a pas résolu tous les problèmes pour toutes les applications mais on a les moyens de progresser dans cette voie. Il faut nous souhaiter bon courage.

\section{Bibliographie}

1 H.A. Macleod, "THIN FILM OPTICAL FILTERS," Adam Hilger, Bristol, 1986.

2 M. Liddell, "COMPUTER AIDED TECHNIQUES FOR DESIGN OF MULTILAYER FILTERS", Adam Hilger, Bristol, 1981.

3 A. Thelen, "Equivalent Layers in Multilayer Filters," J. Opt. Soc. Am., 56, 15331538 (1986)

A. Thelen, "DESIGN OF OPTICAL INTERFERENCE COATINGS," (1987)

$4 \quad$ H.K. Pulker, "Coatings on glass," Elsevier (1984)

5 J.M. Elson, "Angle resolved light scattering from composite optical surfaces," Proc. Soc. Photo. Opt. Instrum. Eng. 240, 296 (1980)

6 C. Amra, "Calculs et mesures de diffusion appliqués à l'étude de la rugosité dans les traitements optiques multicouches", J. Optics, 21, 83-98 (1990)

7 A.C. Boccara, D. Fournier, W. Jackson, N.M. Amer, "Sensitive photothermal deflection technique for measuring absorption in optically thin media", Opt. Letters 5, 377 (1980). 
Annexe

\section{Formulaire pour le calcul des propriétés optiques en incidence normale d'un empilement de couches minces}

On écrit les équations de Maxwell

1) Sur un dioptre on écrit les conditions de continuité des composantes tangentielles du champ électrique et magnétique.

On démontre ainsi que :

- à la réflexion sur un dioptre il n'y a pas de changement de longueur d'onde

- les rayons réfléchis et transmis sont dans le plan d'incidence

- $\mathrm{n}_{0} \sin \theta_{0}=\mathrm{n}_{1} \sin \theta_{1} \quad$ (relation de Fresnel)

2) Pour une couche mince

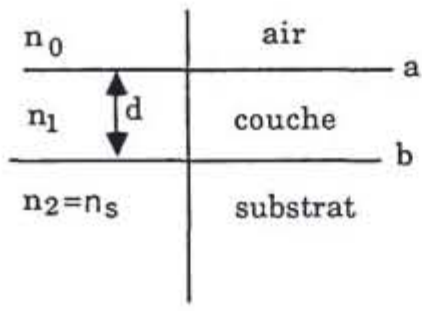

En incidence normale on relie les composantes sur la surface a à celle de la surface b (dioptre substrat couche)

$$
\begin{gathered}
\left(\begin{array}{l}
E_{a} \\
H_{a}
\end{array}\right)=\left(\begin{array}{ll}
\cos \delta & \frac{i \sin \delta}{n_{1}} \\
i n_{1} \sin \delta & \cos \delta
\end{array}\right)\left(\begin{array}{l}
E_{b} \\
H_{b}
\end{array}\right) \\
\operatorname{avec~} \delta=\frac{2 \pi n_{1} d}{\lambda}
\end{gathered}
$$

$\mathrm{y}=\frac{\mathrm{H}_{\mathrm{a}}}{\mathrm{E}_{\mathrm{a}}}$

sur la surface du substrat $n_{\mathrm{s}}: \quad \frac{\mathrm{H}_{\mathrm{b}}}{\mathrm{E}_{\mathrm{b}}}=\mathrm{n}_{\mathrm{s}}$

On pose $y=\frac{C}{B} \quad$ avec $\left(\begin{array}{l}B \\ C\end{array}\right)=\left(\begin{array}{ll}\cos \delta & \frac{i \sin \delta}{n_{1}} \\ i n_{1} \sin \delta & \cos \delta\end{array}\right)\left(\begin{array}{l}1 \\ n_{2}\end{array}\right)$

Le coefficient de réflexion pour l'amplitude est :

$$
\begin{aligned}
& \rho=\frac{n_{0}-y}{n_{0}+y} \\
& R=\left(\frac{n_{0}-y}{n_{0}+y}\right)\left(\frac{n_{0}-y}{n_{0}+y}\right)^{*} \quad \text { S'il n'y a pas d'absorption } T=1-R
\end{aligned}
$$

3) Pour un empilement de couches. Au lieu d'une matrice on a un produit de matrices (attention à l'ordre des matrices !)

$$
\begin{aligned}
n_{0}\left|n_{1}\right| n_{2}\left|n_{3}\right| n_{4}\left|n_{5}\right| \begin{array}{l}
n_{s} \\
\left(=n_{6}\right)
\end{array} \\
\text { On calcule }\left(\begin{array}{l}
B \\
C
\end{array}\right)=\prod_{r=1}^{P}\left[\begin{array}{ll}
\cos \delta_{r} & \frac{i \sin \delta_{r}}{n_{r}} \\
i n_{r} \sin \delta_{r} & \cos \delta_{r}
\end{array}\right] \quad\left(\begin{array}{l}
1 \\
n_{s}
\end{array}\right) \text { avec } \delta_{r}=\frac{2 \pi n_{r} d_{r}}{\lambda}
\end{aligned}
$$

